CERN-TH.7379/94

hep-th/9408033

\title{
Superstrings from Hamiltonian Reduction
}

\author{
A. Boresch, K. Landsteiner, W. Lerche, A. Sevrin \\ CERN, Geneva, Switzerland
}

\begin{abstract}
In any string theory there is a hidden, twisted superconformal symmetry algebra, part of which is made up by the BRST current and the anti-ghost. We investigate how this algebra can be systematically constructed for strings with $N-2$ supersymmetries, via quantum Hamiltonian reduction of the Lie superalgebras $\operatorname{csp}(N \mid 2)$. The motivation is to understand how one could systematically construct generalized string theories from superalgebras. We also briefly discuss the BRST algebra of the topological string, which is a doubly twisted $N=4$ superconformal algebra.
\end{abstract}

CERN-TH.7379/94

July 1994 


\section{Introduction}

It is known that the BRST structure of (critical and non-critical) bosonic strings can be characterized in terms of a twisted $N=2$ superconformal algebra [1], that of superstrings in terms of an $N=3$ algebra and that of $W_{n}$-strings in terms of $N=2 W_{n}$-algebras [2], etc. In terms of a given superconformal algebra, the nilpotent BRST operator is nothing but one of the supercharges,

$$
\mathcal{Q}_{B R S T} \equiv G_{0}^{+}=\oint d z(c T+\ldots)
$$

such that the fundamental relation $T(z)=\left\{\mathcal{Q}_{B R S T}, b(z)\right\}$ is part of an $N=2$ sub-algebra (the anti-ghost is just the conjugate supercurrent, $G^{-}(z)=b(z)$ ). The above expression represents a very specific realization of such superconformal algebras, and it was shown in [2, 4] that for ordinary bosonic and $W_{n}$-strings, this kind of realization can indeed be systematically obtained via quantum Hamiltonian reduction from WZW models based on $s \ell(n \mid n-1)$. (Note that there exists a different, complementary way of characterizing the BRST structure in terms of superconformal algebras, initiated in [3]. These BRST algebras seem to be much harder to obtain from Hamiltonian reduction, if at this is all possible, because they involve bosonization of the $\beta, \gamma$ systems.)

Embedding of string theories into WZW models allows to analyze their BRST structure by Lie algebraic methods, and more importantly, opens up the possibility of classifying string theories in terms of superalgebras. Since the Hamiltonian reduction of $s \ell(2 \mid 1)$ yields the ordinary bosonic string, one may expect that a very large class extensions of the bosonic string are determined by the embeddings of $s \ell(2 \mid 1)$ into arbitrary superalgebras. However, what really makes a string model is certainly not just any superconformal algebra obtained in this way: in order to define a string theory, one also must require a particular realization of the algebra, namely one where one of the supercharges has the form (1.1).

The existence of such realizations is a priori not obvious, and it is the purpose of this paper to shed some light on this problem of constructing general string BRST operators from superalgebras. We will primarily focus on how superstrings with $N-2$ supersymmetries can be obtained via quantum Hamiltonian reduction from the superalgebras

\footnotetext{
${ }^{1}$ Note that $G^{+}(z)$ will in general explicitly contain the Liouville field or some matter fields, so that it is not really covariant. However, since these fields appear only in total derivative terms, this is irrelevant for the classification of BRST operators.
} 
$\operatorname{osp}(N \mid 2)$. It turns out that in comparison to $W_{n}$-strings (associated with $s \ell(n \mid n-1)$ ), the situation is much more involved here, in that various complications occur. To our knowledge, some of these complications in the Hamiltonian reduction and their resolution have so far not been treated in the literature.

We will start in section 2 with the ordinary $N=1$ superstring, for which we will be most explicit since it already displays the relevant features. In the following section, we will then consider the Hamiltonian reduction leading to the $N=2$ string, after having first constructed its twisted $N=4$ symmetry algebra (such an algebra was constructed so far only for the critical $N=2$ string [5, 6]). In section 4 we discuss the generalization to arbitrary $N$-extended superstrings, and in section 5 we will consider topological strings. Even though we find an analogous, but now doubly twisted $N=4$ superconformal symmetry in these theories, we did not quite succeed to obtain the relevant realization of this algebra from Hamiltonian reduction.

\section{$2 \mathrm{~N}=1$ Superstring}

The idea of quantum Hamiltonian reduction is to first choose an embedding of $s \ell(2)$ into a given Lie (super-)algebra and an element of the Cartan sub-algebra (that necessarily contains the Cartan generator of the $s \ell(2))$. This Cartan element will give rise to a splitting of the Lie algebra into sub-algebras with positive, zero and negative grades. Imposing first-class constraints on the negative-grade part induces a gauge symmetry of the associated WZW-model. In particular, one always constrains the lowering operator of the $s \ell(2)$ to be a constant, in order to ensure the existence of a Virasoro generator. The other non-trivial elements of the BRST cohomology of this gauged WZW-model then form an extension of the Virasoro algebra [7].

Before applying these ideas to $\operatorname{osp}(3 \mid 2)$, we first give a brief description of its current algebra (the general conventions for $\operatorname{osp}(N \mid 2)$ current algebras are summarized in appendix A). The bosonic part of $\operatorname{ssp}(3 \mid 2)$ consists of an $s \ell(2)$ current algebra at level $\kappa$ and an $s o(3)$ current algebra at level $-2 \kappa$. It is convenient for our purposes to choose a basis of the $s o(3)$ sub-algebra by $J^{ \pm}=\left(J^{1} \pm i J^{2}\right), J^{0}=i J^{3}$. The fermionic part trans-

forms according to the spin- $\frac{1}{2}$ representation of $s \ell(2)$ and as a vector under $s o(3)$. We will denote the fermionic currents by $j^{a b}$ with $a \in\{+,-\}$ and $b \in\{+, 0,-\}$. The generators 
of the Lie superalgebra are denoted by $e_{ \pm}, e_{0}, t_{ \pm}^{J}, t_{0}^{J}$ and $t_{a b}$.

A natural choice for the $s \ell(2)$ embedding is to take just the bosonic $s \ell(2)$ sub-algebra of $\operatorname{osp}(3 \mid 2)$. It was shown previously [8] that such a quantum Hamiltonian reduction of $\operatorname{osp}(N \mid 2)$ gives rise to the standard $N$-extended superconformal algebra, if one uses the gradation corresponding to the Cartan element $E^{0}$. Taking over the general line of argumentation of [2], we expect that different choices of gradations will result in different realizations of the same extended Virasoro algebra. Indeed we will find that if we choose instead the Cartan element $E^{0}+J^{0}$ for the gradation, we precisely obtain the non-standard realization of the $N=3$ superconformal algebra that represents the BRST algebra of the $N=1$ superstring.

There are, however, differences and new features as compared to the $\operatorname{sl}(n \mid n-1)$ reductions, which describe the $W_{n}$ strings. First, in [2, 4] the possibility of having various, genuinely different free-field realizations of the same superconformal algebra was attributed to the possibility of gauging different Borel subgroups, which for $s \ell(n \mid n-1)$ happens to be equivalent to choosing different gradations. However, in the present context of $\operatorname{ssp}(N \mid 2)$, it appears that the realizations we are after cannot be obtained by simply gauging different Borel subgroups. In other words, we find that choosing different gradations is a more general method than gauging different Borel sub-algebras.

In addition, the superconformal algebras that one obtains by Hamiltonian reduction will always be non-linearly [9] generated, whereas the twisted $N=3$ algebra of the $N=1$ string (and similarly the twisted $N=4$ algebra of the $N=2$ string discussed in chapter 3 ) is linear. This is because the supercharges that represent the BRST-current and the anti-ghost are supposed to be nilpotent, and this is only the case for the linear form of the $N$-extended superconformal algebras. Moreover, the numbers of the free fermions that we get from the reduction will a priori be less than the number of the fields of the string model. Therefore we have to adjoin additional fermions in a rather special way to linearize the algebra and to obtain the proper free field realization that can be attributed to the string theory; this is essentially the inverse of the fermion-decoupling procedure of [10]. We will expand on these points later on.

Adopting the Cartan element $E^{0}+J^{0}$, we have the following grades of the currents: 


\begin{tabular}{|c||c|c|c||c|c|c||c|c|c|c|c|c|}
\hline & $E^{+}$ & $E^{0}$ & $E^{-}$ & $J^{+}$ & $J^{0}$ & $J^{-}$ & $j^{++}$ & $j^{+0}$ & $j^{+-}$ & $j^{-+}$ & $j^{-0}$ & $j^{--}$ \\
\hline$E^{0}$ & 1 & 0 & -1 & 0 & 0 & 0 & $1 / 2$ & $1 / 2$ & $1 / 2$ & $-1 / 2$ & $-1 / 2$ & $-1 / 2$ \\
\hline$J^{0}$ & 0 & 0 & 0 & 1 & 0 & -1 & 1 & 0 & -1 & 1 & 0 & -1 \\
\hline$E^{0}+J^{0}$ & 1 & 0 & -1 & 1 & 0 & -1 & $3 / 2$ & $1 / 2$ & $-1 / 2$ & $1 / 2$ & $-1 / 2$ & $-3 / 2$ \\
\hline
\end{tabular}

A consistent set of first-class constraints is given by $\Phi^{\alpha}=0$, where

$$
\begin{aligned}
\Phi_{E}^{-} & =E^{-}-\frac{\kappa}{2}, \\
\Phi_{J}^{-} & =J^{-}-\lambda, \\
\Phi^{+-} & =j^{+-}-\psi, \\
\Phi^{-0} & =j^{-0}-\tau-\bar{\psi} \lambda, \\
\Phi^{--} & =j^{--} .
\end{aligned}
$$

The OPE's for the auxiliary fields are

$$
\psi(z) \bar{\psi}(w)=-\frac{1}{(z-w)}, \tau(z) \tau(w)=-\frac{1}{8} \frac{\kappa}{(z-w)}, \lambda(z) \bar{\lambda}(w)=\frac{1}{(z-w)} .
$$

The auxiliary field $\tau$ is necessary in order to make the constraints first-class. On the other hand, the origin of the other auxiliary fields $\psi, \bar{\psi}, \lambda, \bar{\lambda}$ is quite different (the latter does not even appear in the constraints). To see this, note that (2.1) would still form a closed set of first class constraints even when setting $\psi, \bar{\psi}$, and $\lambda$ to zero. However, this would rather correspond to a different $s \ell(2)$ embedding and thus would lead, as a consequence, to a different extended superconformal algebra.

But we really want the embedding being given by $E^{+}, E^{0}, E^{-}$. From general theorems about quantum Hamiltonian reduction, we know that to each highest weight of the embedded $s \ell(2)$, there corresponds a generator of the extended Virasoro algebra. Hence we cannot constrain $J^{-}$and $j^{+-}$to be zero and simultaneously have that these fields survive in the highest weight gauge. The resolution of this problem becomes more clear by writing down a Lagrangian for the constrained WZW-model of $o s p(3 \mid 2)$ :

$$
S=S_{W Z W}[g]+\frac{1}{2 \pi} \int d^{2} z \operatorname{str}(\bar{A} \Phi)-\frac{1}{2 \pi} \int d^{2} z(\bar{\lambda} \bar{\partial} \lambda+\bar{\psi} \bar{\partial} \psi)-\frac{2}{\kappa \pi} \int d^{2} z \tau \bar{\partial} \tau
$$

It is gauge invariant provided the fields transform according to

$$
\delta \tau=\frac{\kappa}{2} \eta^{+0}
$$




$$
\begin{aligned}
\delta \psi=\eta^{+0} \lambda, & \delta \bar{\psi}=2 \eta^{+-}, \\
\delta \lambda=0, & \delta \bar{\lambda}=2 \eta_{J}^{+}+\eta^{+0} \bar{\psi}, \\
\delta \bar{A}=\bar{\partial} \eta+[\bar{A}, \eta], & \delta J=\partial J+[J, \eta] .
\end{aligned}
$$

Here, $\bar{A}$ is the gauge field that arises as Lagrange multiplier for imposing the constraints, $J=\frac{\kappa}{2} \partial g g^{-1}$ denotes the currents of the WZW-model and $\eta$ is the gauge parameter which takes values only in the positively graded part of $\operatorname{ssp}(3 \mid 2)$. Several features of this lagrangian are remarkable. First we see that despite we do not need the field $\bar{\lambda}$ for writing down the constraints it is nessecary for having gauge invariance. Note also that $\psi$, which corresponds to the the highest weight $j^{+-}$, transforms non-trivially. Nevertheless, in the highest weight gauge we have $\eta^{+0}=-\frac{2}{\kappa} \tau$ and therefore $\delta \psi=-\frac{2}{\kappa} \tau \lambda$, which shows that $\psi$ is indeed non-zero and corresponds to a generator of the $N=3$ algebra.

To quantize the action (2.3), we use the BRST-formalism and introduce ghosts and anti-ghosts as follows:

$$
\begin{aligned}
& C=c e_{+}+c^{J} t_{+}^{J}+\gamma^{-+} t_{-+}+\gamma^{+0} t_{+0}+\gamma^{++} t_{++}, \\
& B=b e_{-}+b^{J} t_{-}^{J}+\beta^{+-} t_{+-}+\beta^{-0} t_{-0}+\beta^{--} t_{--},
\end{aligned}
$$

The BRST-transformations are:

$$
s B=D \quad, \quad s \bar{A}=-\bar{\partial} C-[\bar{A}, C]
$$

We impose the light-cone gauge by adding to (2.3)

$$
S_{g . f .}=s \frac{1}{2 \pi} \int d^{2} z B \bar{A}=\frac{1}{2 \pi} \int d^{2} z(D \bar{A}+B \bar{\partial} C+\bar{A}\{B, C\}) .
$$

From this we can read off the OPE's for the ghosts

$$
\begin{array}{r}
b(z) c(w)=-b^{J}(z) c^{J}(w)=-\beta^{+-}(z) \gamma^{-+}(w)=\beta^{--}(z) \gamma^{++}(w)=, \\
2 \beta^{-0}(z) \gamma^{+0}(w)=\frac{1}{(z-w)} .
\end{array}
$$

Integrating out the gauge field then yields

$$
D=s B=\hat{\Phi}
$$

where $\hat{\Phi}$ denotes the substitution of $J$ by $\hat{J}=J+J_{g h}$ and $J_{g h}=\frac{1}{2}\{B, C\}$. Explicitly, the ghost currents are

$$
E_{g h}^{+}=-\frac{1}{2} \beta^{+-} \gamma^{++}
$$




$$
\begin{aligned}
E_{g h}^{0} & =-\frac{1}{2} b c+\frac{1}{4} \beta^{+-} \gamma^{-+}+\frac{1}{2} \beta^{-0} \gamma^{+0}+\frac{1}{4} \beta^{--} \gamma^{++} \\
E_{g h}^{-} & =\frac{1}{2} \beta^{--} \gamma^{-+} \\
J_{g h}^{+} & =-\frac{1}{2} \beta^{-0} \gamma^{++} \\
J_{g h}^{0} & =-\frac{1}{2} b^{J} c^{J}-\frac{1}{4} \beta^{+-} \gamma^{-+}+\frac{1}{2} \beta^{-0} \gamma^{+0}, \\
J_{g h}^{-} & =-\frac{1}{2} \beta^{--} \gamma^{+0} \\
j_{g h}^{+0} & =\frac{1}{4} b^{J} \gamma^{++}-\frac{1}{4} \beta^{+-} c^{J}+\frac{1}{2} \beta^{-0} c \\
j_{g h}^{+-} & =-\frac{1}{2} b^{J} \gamma^{+0}+\frac{1}{2} \beta^{--} c \\
j_{g h}^{-+} & =\frac{1}{2} b \gamma^{++}+\frac{1}{2} \beta^{-0} c^{J}, \\
j_{g h}^{-0} & =\frac{1}{4} b \gamma^{+0}+\frac{1}{4} b^{J} \gamma^{-+}-\frac{1}{4} \beta^{--} c^{J},
\end{aligned}
$$

Eq. (2.9) shows that the anti-ghosts and the constraint currents form BRST doublets and thus decouple from the BRST cohomology. The ghost contributions to the total currents $\hat{J}$ modify the central extensions of the algebra. In particular, we have the following modified central terms for the Cartan currents:

$$
\begin{gathered}
\hat{E}^{0}(z) \hat{E}^{0}(w)=\frac{(1+2 \kappa)}{16} \frac{1}{(z-w)^{2}}, \\
\hat{J}^{0}(z) \hat{J}^{0}(w)=-\frac{(1+2 \kappa)}{16} \frac{1}{(z-w)^{2}},
\end{gathered}
$$

It is now straightforward to write down the BRST operator:

$$
\begin{aligned}
\mathcal{Q}_{B R S T}= & \frac{1}{2 \pi i} \oint d^{2} z\left[c\left(E^{-}+\frac{1}{2} E_{g h}^{-}-\frac{\kappa}{2}\right)-c^{J}\left(J^{-}+\frac{1}{2} J_{g h}^{-}-\lambda\right)+\gamma^{-+}\left(j^{+-}+\right.\right. \\
& \left.\left.+\frac{1}{2} j_{g h}^{+-}-\psi\right)-2 \gamma^{+0}\left(j^{-0}+\frac{1}{2} j_{g h}^{-0}-\tau-\frac{1}{4} \bar{\psi} \lambda\right)-\gamma^{++} j^{--}\right]
\end{aligned}
$$

which can be split it into the three following pieces:

$$
\begin{aligned}
\mathcal{Q}_{0}= & \frac{1}{2 \pi i} \oint d^{2} z\left(-\frac{\kappa}{2} c+c^{J} \lambda\right), \\
\mathcal{Q}_{1}= & \frac{1}{2 \pi i} \oint d^{2} z\left(-\gamma^{-+} \psi+2 \gamma^{+0} \tau+\frac{1}{2} 2 \gamma^{+0} \bar{\psi} \lambda\right), \\
\mathcal{Q}_{2}= & \frac{1}{2 \pi i} \oint d^{2} z\left[c\left(E^{-}+\frac{1}{2} E_{g h}^{-}\right)-c^{J}\left(J^{-}+\frac{1}{2} J_{g h}^{-}\right)+\gamma^{-+}\left(j^{+-}+\frac{1}{2} j_{g h}^{+-}\right)-\right. \\
& \left.2 \gamma^{+0}\left(j^{-0}+\frac{1}{2} j_{g h}^{-0}\right)-\gamma^{++} j^{--}\right],
\end{aligned}
$$


where $\mathcal{Q}_{0}^{2}=\mathcal{Q}_{2}^{2}=0$ and $\mathcal{Q}_{1}^{2}+\left\{\mathcal{Q}_{0}, \mathcal{Q}_{2}\right\}=0$. This shows that the techniques developed in [7] can be fully taken over. In particular, the spectral sequence techniques apply and we can define the quantum Miura transformation as the truncation of the generators of the cohomology of (2.12) to the zero-grade fields, which are given by the the Cartan generators of $\operatorname{osp}(3 \mid 2)$ and the auxiliary fields. This method guarantees that we end up with a free-field realization of the non-linear $N=3$ superconformal algebra. Note that this is specifically due to our gradation $E^{0}+J^{0}$ (for the usual gradation $E^{0}$ [8], the whole $s o(N)$ sub-algebra of $\operatorname{osp}(N \mid 2)$ has grade zero and thus does not get bosonized).

Using the methods of [7], where we use the filtration of $\mathcal{Q}_{B R S T}$ given by (2.13), we can compute the non-trivial elements of the cohomology by starting with the total currents that are $s \ell(2)$ highest weights. In this way, we find for the stress tensor:

$$
\begin{aligned}
T= & \frac{4 \kappa}{1+2 \kappa}\left(\hat{E}^{+}+\frac{2}{\kappa} \hat{E}^{0^{2}}-\partial \hat{E}^{0}-\frac{2}{\kappa} \hat{J}^{0^{2}}+\alpha \partial \hat{J}^{0}-\frac{1-2 \alpha}{8 \kappa} \partial \lambda \bar{\lambda}+\right. \\
& +\frac{1+4 \kappa+2 \alpha \kappa}{8 \kappa} \lambda \partial \bar{\lambda}+\frac{1+2 \kappa}{k^{2}} \tau \partial \tau+\frac{1+3 \kappa+\alpha \kappa}{4 \kappa} \psi \partial \bar{\psi}+\frac{1+\alpha}{4} \partial \psi \bar{\psi}- \\
& \left.-\frac{4}{\kappa} \hat{j}^{+0} \tau+\frac{2}{\kappa} \hat{j}^{-+} \psi-\frac{2}{\kappa} \hat{J}^{+} \lambda+\frac{1}{\kappa} \hat{j}^{+0} \lambda \psi\right)
\end{aligned}
$$

and for the generators of the so(3) Kac-Moody algebra:

$$
\begin{aligned}
K^{+}= & 4\left(\hat{J}+\frac{2}{\kappa} \hat{j}^{-+} \tau-\frac{1}{2 \kappa} \hat{j}^{-+} \lambda \bar{\psi}-\frac{1}{\kappa} \hat{E}^{0} \bar{\psi} \tau-\frac{1}{2} \hat{J}^{0} \bar{\lambda}-\frac{1}{2} \hat{j}^{+0} \bar{\psi}-\frac{1}{\kappa} \hat{J}^{0} \bar{\psi} \tau+\right. \\
& \left.+\frac{1}{2 \kappa} \hat{j}^{-+} \bar{\psi} \lambda-\frac{1+2 \kappa}{8} \partial \bar{\lambda}-\frac{1+2 \kappa}{4} \partial \bar{\psi} \tau-\frac{1}{16} \bar{\lambda}^{2} \lambda-\frac{1}{8} \bar{\lambda} \psi \bar{\psi}\right), \\
K^{3}= & 4\left(\hat{J}^{0}+\frac{1}{4} \bar{\lambda} \lambda-\frac{1}{4} \bar{\psi} \psi\right), \\
K^{-}= & 4 \lambda .
\end{aligned}
$$

In addition, we get the following three supercurrents:

$$
\begin{aligned}
G^{+}= & \frac{4 i \sqrt{2}}{\sqrt{1+2 \kappa}}\left(\hat{j}^{++}-\frac{(1+2 \kappa)^{2}}{16 \kappa} \partial^{2} \bar{\psi}+\frac{1}{2} \hat{E}^{+} \bar{\psi}-\frac{2}{\kappa} \hat{J}^{+} \tau-\frac{1}{2} \hat{j}^{+0} \bar{\lambda}-\right. \\
& -\hat{E}^{0} \hat{j}^{-+}+\frac{2}{\kappa} \hat{J}^{0} \hat{j}^{-+}+\frac{1}{\kappa} \hat{E}^{0^{2}} \bar{\psi}+\frac{1}{\kappa} \hat{J}^{0^{2}} \bar{\psi}-\frac{1}{2} \partial E^{0} \bar{\psi}-\frac{1}{2} \partial \hat{J}^{0} \bar{\psi}+ \\
& +\frac{1+2 \kappa}{8 \kappa} \psi \partial \bar{\psi} \bar{\psi}+\frac{1}{\kappa} \hat{j}^{+0} \bar{\psi} \tau-\frac{1}{\kappa} \hat{j}^{-+} \bar{\psi} \psi+\frac{1}{2 \kappa} \hat{j}^{+0} \bar{\lambda} \lambda- \\
& -\frac{1+2 \kappa}{16 \kappa} \partial \bar{\psi} \bar{\lambda} \lambda-\frac{1}{16} \lambda^{2} \psi+\frac{1}{\kappa} \hat{E}^{0} \bar{\lambda} \tau-\frac{1}{4 \kappa} \hat{J}^{0} \bar{\psi} \bar{\lambda} \lambda- \\
& -\frac{1+2 \kappa}{4 \kappa} \bar{\lambda} \partial \tau-\frac{1}{4 \kappa} \hat{E}^{0} \bar{\psi} \lambda \bar{\lambda}-\frac{1}{8 \kappa} \bar{\lambda}^{2} \lambda \tau+\frac{1}{4 \kappa} \bar{\psi} \psi \bar{\lambda} \tau-\frac{1}{4 \kappa} \partial \bar{\psi} \hat{E}^{0}- \\
& \left.-\frac{3+4 \kappa}{4 \kappa} \bar{\psi} \hat{J}^{0}+\partial \hat{j}^{-+}\right),
\end{aligned}
$$




$$
\begin{aligned}
G^{3}= & \frac{4 i \sqrt{2}}{\sqrt{1+2 \kappa}}\left(\hat{j}^{+0}-\frac{1}{\kappa} \hat{j}^{-+} \lambda+\frac{1+2 \kappa}{8 \kappa} \partial \bar{\psi} \lambda+\frac{1}{4} \bar{\lambda} \psi-\frac{2}{\kappa} \hat{E}^{0} \tau+\frac{1}{2 \kappa} \hat{J}^{0} \bar{\psi} \lambda+\right. \\
& \left.+\frac{1+2 \kappa}{2 \kappa} \partial \tau+\frac{1}{2 \kappa} \hat{E}^{0} \bar{\psi} \lambda+\frac{1}{2 \kappa} \bar{\lambda} \lambda \tau-\frac{1}{2 \kappa} \bar{\psi} \psi \tau\right), \\
G^{-}= & \frac{4 i \sqrt{2}}{\sqrt{1+2 \kappa}}\left(\psi+\frac{2}{\kappa} \tau \lambda\right) .
\end{aligned}
$$

From these expressions we get a free-field realization of the $N=3$ superconformal algebra in nonlinear form, by taking only the zero grade part of each generator. However, a simple count of the number of fields shows that there is one fermion missing to give the field content of the $N=1$ string (assuming that the matter sector is realized in terms of one boson and one fermion). Of course, this is not surprising since the non-linear form of the algebra is well-known to arise by factorization of a single fermion [10].

Note also that in the expression for the stress tensor there is a free parameter $\alpha$, which determines the conformal weights of $K^{+}, K^{-}, G^{+}, G^{-}$, and thus serves as a twist parameter. If we choose the value $\alpha=1+\frac{1}{\kappa}$, we get conformal weight $1 / 2$ for $K^{+}, 3 / 2$ for $K^{-}, 1$ for $G^{+}$and 2 for $G^{-}$. This choice is motivated by the string representation, where $G^{-}$is the anti-ghost. The resulting central charge of the stress tensor is then $-1 / 2$. Since in a string theory the central extension of the Virasoro algebra should vanish, this is further indication of the missing of one fermionic degree of freedom. Therefore, we adjoin a free fermion $\rho$ with OPE

$$
\rho(z) \rho(w)=-\frac{(1+2 \kappa)}{(z-w)} .
$$

We can now linearize the algebra by redefining the generators

$$
\begin{aligned}
T_{\text {lin }} & =T+\frac{1}{2(1+2 \kappa)} \rho \partial \rho, \\
G_{\text {lin }}^{a} & =G^{a}+\frac{1}{1+\kappa} \rho K^{a} .
\end{aligned}
$$

This indeed results in a free-field realization of the linear $N=3$ algebra (cf., (B.2)). However, the generator $G^{-}$is not yet precisely of the desired form, for which $G^{-} \propto \psi$. Rather, we obtain the expression

$$
G^{-}=\frac{4 i \sqrt{2 \kappa}}{\sqrt{1+2 \kappa}}\left(\psi+\frac{2}{\kappa} \tau \lambda\right)-\frac{4}{1+2 \kappa} \rho \lambda .
$$

From this we can infer that we need, in addition, to perform a similarity transformation

$$
\tilde{T}=S T S^{-1},
$$




$$
\begin{aligned}
\tilde{G}^{a} & =S G^{a} S^{-1}, \\
\tilde{K}^{a} & =S K^{a} S^{-1}, \\
F & =S \rho S^{-1},
\end{aligned}
$$

where

$$
S=\exp \left[\frac{1}{2 \pi i} \oint d z\left(\frac{2}{\kappa} \tau \lambda \bar{\psi}-\frac{i}{\sqrt{2 \kappa(1+2 \kappa)}} \rho \lambda \bar{\psi}\right)\right] .
$$

Furthermore, we bosonize the Cartan currents as follows:

$$
\begin{aligned}
\hat{E}^{0} & =i \frac{\sqrt{1+2 \kappa}}{4} \partial \varphi_{1} \quad, \quad \hat{J}^{0}=\frac{\sqrt{1+2 \kappa}}{4} \partial \varphi_{2}, \\
\varphi_{k}(z) \varphi_{l}(w)=-\delta_{k l} \ln (z-w), &
\end{aligned}
$$

and rescale the remaining fields according to

$$
\begin{aligned}
\rho \rightarrow \frac{1}{\sqrt{1+2 \kappa}} \rho \quad, \quad \tau \rightarrow \frac{2 \sqrt{2}}{\sqrt{\kappa}} \tau, \\
\psi \rightarrow \frac{2 \sqrt{2 \kappa}}{\sqrt{1+2 \kappa}} \psi \quad, \quad \bar{\psi} \rightarrow-\frac{\sqrt{1+2 \kappa}}{2 \sqrt{2 \kappa}} \bar{\psi}, \\
\lambda \rightarrow-2 i \lambda \quad, \quad \bar{\lambda} \rightarrow \frac{i}{2} \bar{\lambda} .
\end{aligned}
$$

Then, after all these manipulations, we finally arrive at the following form for the Miuratransformed linearized generators:

$$
\begin{aligned}
\tilde{T}= & -\frac{1}{2}\left(\partial \varphi_{1}\right)^{2}-\frac{1}{2}\left(\partial \varphi_{2}\right)^{2}+\frac{3}{2} \lambda \partial \bar{\lambda}+\frac{1}{2} \partial \lambda \bar{\lambda}-2 \psi \partial \bar{\psi}-\partial \psi \bar{\psi}+ \\
& +\frac{1}{2} \rho \partial \rho+\frac{1}{2} \tau \partial \tau-\frac{i \kappa}{\sqrt{1+2 k}} \partial^{2} \varphi_{1}+\frac{1+\kappa}{\sqrt{1+2 k}} \partial^{2} \varphi_{2}, \\
\tilde{G}^{+}= & 2 i\left(\overline { \psi } \left(\frac{1}{2}\left(\partial \varphi_{1}\right)^{2}+\frac{1}{2}\left(\partial \varphi_{1}\right)^{2}+\frac{i \kappa}{\sqrt{1+2 \kappa}} \partial^{2} \varphi_{1}-\frac{1+\kappa}{\sqrt{1+2 k}} \partial^{2} \varphi_{2}-\frac{3}{2} \lambda \partial \bar{\lambda}-\right.\right. \\
& \left.-\frac{1}{2} \partial \lambda \bar{\lambda}-\frac{1}{2} \rho \partial \rho-\frac{1}{2} \tau \partial \tau\right)+\bar{\lambda}\left(\frac{1}{2} \partial \varphi_{1} \tau+\frac{1}{2} \partial \varphi_{2} \rho+\frac{i \kappa}{\sqrt{1+2 k}} \partial \tau-\right. \\
& \left.-\frac{1+\kappa}{\sqrt{1+2 \kappa}} \partial \rho\right)-\frac{1}{2}\left(\frac{3}{2} \bar{\psi} \lambda \partial \bar{\lambda}+\frac{1}{2} \bar{\psi} \partial \lambda \bar{\lambda}-\bar{\psi} \psi \partial \bar{\psi}+\frac{3}{2} \bar{\lambda} \lambda \partial \bar{\psi}+\right. \\
& \left.+\frac{1}{2} \bar{\lambda}^{2} \psi+\bar{\psi} \bar{\lambda} \partial \lambda\right)+\frac{\sqrt{1+2 k}}{2} \partial(\bar{\lambda} \rho)+\frac{1+2 k}{2} \partial^{2} \bar{\psi}+\frac{1}{4} \partial(\bar{\lambda} \lambda \bar{\psi})+ \\
& \left.+\sqrt{1+2 \kappa} \partial\left(\varphi_{2} \bar{\psi}\right)\right), \\
\tilde{G}^{3}= & \partial \varphi_{1} \tau+\partial \varphi_{2} \rho+3 \lambda \partial \bar{\psi}+2 \partial \lambda \bar{\psi}+\psi \bar{\lambda}+\frac{2 i \kappa}{\sqrt{1+2 \kappa}} \partial \tau-\frac{2+2 \kappa}{\sqrt{1+2 \kappa}} \partial \rho, \\
\tilde{G}^{-}= & 2 i \psi,
\end{aligned}
$$




$$
\begin{aligned}
\tilde{K}^{+}= & 2 i\left(-\frac{1}{2} \bar{\psi} \partial \varphi_{1} \tau-\frac{1}{2} \bar{\psi} \partial \varphi_{2} \rho+\frac{1}{2} \bar{\psi} \lambda \partial \bar{\psi}+\frac{1}{2} \bar{\psi} \bar{\lambda}+\frac{i \kappa}{\sqrt{1+2 \kappa}} \bar{\psi} \partial \varphi_{1}+\right. \\
& +\frac{\kappa}{\sqrt{1+2 \kappa}} \bar{\psi} \partial \varphi_{2}-\frac{\sqrt{1+2 \kappa}}{2} \partial \varphi_{2} \bar{\lambda}+\frac{1}{4} \bar{\lambda}^{2} \lambda+\frac{\sqrt{1+2 \kappa}}{2} \partial \bar{\psi} \rho+ \\
& \left.+\frac{1+2 \kappa}{2} \partial \bar{\lambda}\right), \\
\tilde{K}^{3}= & -\psi \bar{\psi}+\lambda \bar{\lambda}+\sqrt{1+2 \kappa} \partial \varphi_{2}, \\
\tilde{K}^{-}= & 2 i \lambda \\
F= & \lambda \bar{\psi}+\sqrt{1+2 \kappa} \rho .
\end{aligned}
$$

These formulas correspond exactly to the generators of the hidden $N=3$ superconformal algebra of the $N=1$ superstring as found in [2]. We just need to identify $\varphi_{1}$ and $\tau$ with the matter system, and $\varphi_{2}$ and $\rho$ with the Liouville system. Moreover, we see that the diffeomorphism ghost of the superstring is represented by $\bar{\psi}$, the anti-ghost by $\psi$, and their bosonic superpartners by $\bar{\lambda}$ and $\lambda$, respectively. This means that $G^{+}$is indeed the $N=1$ string BRST operator, $K^{+}$its superpartner with respect to the $N=1$ supercharge, $G^{3}$, and $K^{3}$ is the ghost number current. Finally, note that the Kac-Moody level $\kappa$ is related to the matter central charge as follows:

$$
\hat{c}_{m}=5-2\left((1+2 \kappa)+\frac{1}{1+2 \kappa}\right),
$$

where, of course, $\hat{c}_{m}=10$ corresponds to the critical superstring.

\section{$3 \mathrm{~N}=2$ Superstring}

In this section we will generalize the methods discussed above to the $N=2$ superstring [11], [12]. We will first construct the generators of the hidden, twisted $N=4$ superconformal algebra. In fact, such an algebra has already been found in [6], but only for critical $N=2$ strings. We will generalize this to non-critical $N=2$ strings, where the conformal anomaly is cancelled by the $N=2$ Liouville system.

To begin, we would like to make some general remarks about the twisted $N=4$ algebra. (Our conventions, in which we follow [13], can be found in appendix B.) It is known [14] that $N=4$ algebras come in a lot of varieties, but the most general algebra consists of the energy-momentum tensor $T$, four supercurrents $G_{ \pm}, G_{ \pm K}$, and two commuting $s \ell(2) \mathrm{Kac}-$ Moody algebras at levels $k^{+}$and $k^{-}$whose currents we denote by $A^{ \pm i}$ with $i \in\{+, 3,-\}$. 
Furthermore, there are four fermionic currents of spin $\frac{1}{2}$, denoted by $Q_{ \pm}, Q_{ \pm K}$, plus a $\mathrm{U}(1)$ current $U$. It is possible to redefine the generators such that the spin- $\frac{1}{2}$ currents and the $\mathrm{U}(1)$ current decouple, so that one is left over with the energy momentum tensor, the supercharges and the Kac-Moody currents. This is the non-linear form of the algebra, which is the form that we will get from Hamiltonian reduction of $\operatorname{ssp}(4 \mid 2)$.

Let us first discuss the twisted linear $N=4$ algebra, by noting that there are two separate $N=2$ sub-algebras. We can use one of the two associated $U(1)$ currents to twist the energy-momentum tensor as follows:

$$
\hat{T}=T+\partial M_{+K-K}
$$

where the $N=4$ current $M$ is defined in appendix B. The generators $\hat{T}, G_{ \pm K}$ and $2 M_{+K-K}$ then form a topologically twisted $N=2$ sub-algebra. The other $N=2$ algebra has to have vanishing central charge in order to be consistent with the vanishing conformal anomaly of $\hat{T}$. We can achieve this by redefining

$$
\hat{G}_{ \pm}=G_{ \pm}-\frac{2 k^{ \pm}}{k} \partial Q_{ \pm}
$$

Moreover, the condition for $\hat{G}_{ \pm}$to be primary with respect to $\hat{T}$ forces the levels of the Kac-Moody algebras to be equal: $k^{+}=k^{-}$. In addition, due to these modifications, we also have to redefine the $U(1)$ current

$$
\hat{M}_{+-}=M_{+-}+\frac{U}{2} .
$$

The generators $\hat{T}, \hat{G}_{ \pm}$and $\hat{M}_{+-}$form a $N=2$ superconformal algebra with vanishing central charge. After twisting, the conformal weight of $G_{+K}$ is equal to $1, G_{-K}$ has weight $2, A^{++}$and $A^{--}$have weights $\frac{1}{2}$, and $A^{+-}$and $A^{-+}$have weights $\frac{3}{2}$. Furthermore, $Q_{+K}$ and $Q_{-K}$ have weights 0 and 1 , respectively. The conformal weights of the rest of the generators remain unchanged.

For the construction of this algebra in terms of the field content of a $N=2$ string theory we follow the line of [2]. We do not make any assumption about the specific realization of the matter system. We represent it, generically, by the generators of a $N=2$ superconformal algebra: $T_{m}, G_{m \pm}$ and $J_{m}$, and denote its central charge by $c_{m}$.

The Liouville system is given in terms of a complex scalar field $\phi_{l}$ and a complex spinor $\psi_{l}$ with OPE

$$
\partial \phi_{l}(z) \partial \bar{\phi}_{l}(w)=-\frac{1}{(z-w)^{2}}, \quad \psi_{l}(z) \bar{\psi}_{l}(w)=\frac{1}{z-w}
$$


In terms of these fields we can form an $N=2$ algebra as follows:

$$
\begin{aligned}
J_{l} & =-\psi_{l} \bar{\psi}_{l}-Q \partial \phi_{l}-Q \partial \bar{\phi}_{l}, \\
G_{l+} & =\partial \phi_{l} \bar{\psi}_{l}-Q \partial \bar{\psi}_{l}, \\
G_{l-} & =-\partial \bar{\phi}_{l} \psi_{l}-Q \partial \psi_{l}, \\
T_{l} & =-\partial \phi_{l} \partial \bar{\phi}_{l}-\frac{1}{2} \psi_{l} \partial \bar{\psi}_{l}+\frac{1}{2} \partial \psi_{l} \bar{\psi}_{l}-\frac{Q}{2} \partial^{2} \phi_{l}+\frac{Q}{2} \partial^{2} \bar{\phi}_{l},
\end{aligned}
$$

where we introduced a background charge parameter, $Q$, such that the central charge of the Liouville system is given by $c_{l}=3-6 Q^{2}$.

Since we are dealing with local $N=2$ supersymmetry on the string world sheet, we introduce a $(b, c)$ ghost system for the diffeomorphism symmetry, two bosonic ghost systems $\left(\beta_{ \pm}, \gamma_{\mp}\right)$ for the super-diffeomorphisms, and additional fermionic ghosts $(\eta, \xi)$ with spins $(1,0)$ for the U(1) gauge symmetry. The OPE's of these ghosts are

$$
b(z) c(w)=\beta_{ \pm}(z) \gamma_{\mp}(w)=\eta(z) \xi(w)=\frac{1}{z-w} .
$$

We then find for the $N=2$ algebra in the ghost sector

$$
\begin{aligned}
J_{g}= & -\partial(\eta c)-\beta_{+} \gamma_{-}+\beta_{-} \gamma_{+}, \\
G_{g+}= & \frac{3}{2} \beta_{+} \partial c+\partial \beta_{+} c+\frac{1}{2} \partial \eta \gamma_{+}+\eta \partial \gamma_{+}+\beta_{+} \xi-b \gamma_{+}, \\
G_{g-}= & -\frac{3}{2} \beta_{-} \partial c-\partial \beta_{-} c+\frac{1}{2} \partial \eta \gamma_{-}+\eta \partial \gamma_{-}+\beta_{-} \xi+b \gamma_{-}, \\
T_{g}= & -2 b \partial c-\partial b c+\frac{3}{2} \beta_{+} \partial \gamma_{-}+\frac{1}{2} \partial \beta_{+} \gamma_{-}+\frac{3}{2} \beta \beta_{-} \partial \gamma_{+}+\frac{1}{2} \partial \beta_{-} \gamma_{+}- \\
& -\eta \partial \xi
\end{aligned}
$$

which has a central charge equal to -6 . The total central charge of the $N=2$ string, joining together matter, Liouville fields and ghosts, thus vanishes, provided we fix the Liouville background charge to be $Q= \pm \frac{\sqrt{c_{m}-3}}{\sqrt{6}}$. From the ghosts we can build an additional $N=2$ multiplet of currents

$$
\begin{aligned}
J_{\text {ghost }} & =-\eta c, \\
J_{\text {ghost }} & =\eta \gamma_{+}+\beta_{+} c, \\
J_{\text {ghost }-} & =\eta \gamma_{-}-\beta_{-} c, \\
\mathcal{J}_{\text {ghost }} & =-b c+\beta_{+} \gamma_{-}+\beta_{-} \gamma_{+}-\eta \xi .
\end{aligned}
$$


where the highest-spin component is the ghost number current. The $N=2$ string BRSTcurrent is part of an $N=2$ multiplet as well, which has components:

$$
\begin{aligned}
j_{B R S T}= & c\left(J_{m}+J_{l}+\frac{1}{2} J_{g}\right), \\
j_{B R S T+}= & \gamma_{+}\left(J_{m}+J_{l}+\frac{1}{2} J_{g}\right)-c\left(G_{m+}+G_{l+}+\frac{1}{2} G_{g+}\right), \\
j_{B R S T-}= & \gamma_{-}\left(J_{m}+J_{l}+\frac{1}{2} J_{g}\right)-c\left(G_{m-}+G_{l-}+\frac{1}{2} G_{g-}\right), \\
J_{B R S T}= & c\left(T_{m}+T_{l}+\frac{1}{2} T_{g}\right)+\gamma_{+}\left(G_{m-}+G_{l-}+\frac{1}{2} G_{g-}\right)- \\
& -\gamma_{-}\left(G_{m+}+G_{l+}+\frac{1}{2} G_{g+}\right)+\xi\left(J_{m}+J_{L}+\frac{1}{2} J_{g}\right) .
\end{aligned}
$$

Let us now introduce the modified ghost current

$$
\mathcal{I}=\mathcal{J}_{\text {ghost }}-Q \partial \phi_{l}+Q \partial \bar{\phi}_{l}
$$

and the improved BRST current

$$
\begin{aligned}
\mathcal{I}_{B R S T}= & J_{B R S T}-\frac{1}{2} \partial(c \xi \eta)-\frac{1}{4} \partial\left(c \gamma_{+} \beta_{-}\right)-\frac{1}{4} \partial\left(c \gamma_{-} \beta_{+}\right)+Q^{2} \partial^{2} c+ \\
& +Q \partial\left(c \partial \phi_{l}\right)-Q \partial\left(c \partial \bar{\phi}_{l}\right)+Q \partial\left(\gamma_{+} \psi_{l}\right)-Q \partial\left(\gamma_{-} \bar{\psi}_{l}\right)
\end{aligned}
$$

where the total derivative terms have been chosen such that $\mathcal{I}_{B R S T}$ is a primary field of weight 1 and has regular OPE with itself. Together with the total energy momentum tensor, $T_{t o t}=T_{m}+T_{l}+T_{g}$, and the antighost, $b$, these currents form a topologically twisted $N=2$ algebra with central extension

$$
c=c_{m}-3
$$

Note that the critical $N=2$ string, which has $c_{m}=3$, is mapped onto a twisted $N=4$ algebra with vanishing central charge; it is this case which has previously been discussed in 6 .

In order to make contact with the above-described twisted $N=4$ algebra, we identify

$$
T_{\text {tot }} \leftrightarrow \hat{T}, \mathcal{I}_{B R S T} \leftrightarrow G_{+K}, b \leftrightarrow G_{-K}, \mathcal{I} \leftrightarrow 2 M_{+K-K}
$$

Furthermore, we can identify the original $N=2$ generators of the string, $G_{t o t \pm}=G_{m \pm}+$ $G_{l \pm}+G_{g \pm}$ and $J_{t o t}=J_{m}+J_{l}+J_{g}$, with the twisted currents of the $N=4$ superconformal algebra:

$$
G_{t o t \pm} \leftrightarrow \hat{G}_{ \pm}, \quad J_{t o t} \leftrightarrow \hat{M}_{+-}
$$


Let us briefly sketch how to construct the remaining $N=4$ currents. One reads from the twisted $N=4$ superconformal algebra that

$$
G_{+K}(z) \hat{G}_{+}(w)=\frac{i}{2} \frac{A^{++}(w)}{(z-w)^{2}} .
$$

Similar relations hold for the other generators. With the help of the above-mentioned identifications, one gets the whole twisted $N=4$ algebra, described at the beginning of this section, in terms of the fields of the $N=2$ string. In summary, we find the following expressions for the $s \ell(2)$ Kac-Moody currents in the $(+)$-sector

$$
\begin{aligned}
A^{++}= & i\left(j_{\text {BRST+}}+2 Q \bar{\psi}_{l} \xi+Q \bar{\psi}_{l} \partial c+\frac{1}{2} \beta_{-} \gamma_{+}^{2}-\frac{1}{2} \beta_{+} c \xi+\right. \\
& +\frac{1}{2} \beta_{+} \gamma_{+} \gamma_{-}+\frac{1}{4} \beta_{+} c \partial c+2 Q \gamma_{+} \partial \bar{\phi}_{l}-\frac{1}{2} \gamma_{+} b c+\frac{1}{4} \gamma_{+} c \partial \eta- \\
& \left.-\gamma_{+} \eta \xi+2 Q \partial \bar{\psi}_{l} c+\frac{1}{2} \partial \gamma_{+} c \eta-2 Q^{2} \partial \gamma_{+}\right) \\
A^{+3}= & -\frac{i}{2}\left(J_{\text {tot }}+\mathcal{J}_{\text {ghost }}-\frac{1}{2} \partial j_{\text {ghost }}+2 Q \partial \bar{\phi}_{l}\right) \\
A^{+-}= & -i \beta_{-},
\end{aligned}
$$

and the following expressions for the $s \ell(2)$ Kac-Moody currents in the (-)-sector:

$$
\begin{aligned}
A^{-+}= & -i \beta_{+}, \\
A^{-3}= & -\frac{i}{2}\left(J_{\text {tot }}-\mathcal{J}_{\text {ghost }}-\frac{1}{2} \partial \jmath_{\text {ghost }}+2 Q \partial \phi_{l}\right), \\
A^{--}= & -i\left(j_{B R S T-}-2 Q \psi_{l} \xi+Q \psi_{l} \partial c-\frac{1}{2} \beta_{-} c \xi-\frac{1}{2} \beta_{-} \gamma_{+} \gamma_{-}-\right. \\
& -\frac{1}{4} \beta_{-} c \partial c-\frac{1}{2} \beta_{+} \gamma_{-}^{2}+2 Q \gamma_{-} \partial \phi_{l}+\frac{1}{2} \gamma_{-} b c+\frac{1}{4} \gamma_{-} c \partial \eta+ \\
& \left.+\gamma_{-} \eta \xi+2 Q \partial \psi_{l} c+\frac{1}{2} \partial \gamma_{-} c \eta+2 Q^{2} \partial \gamma_{-}\right) .
\end{aligned}
$$

Furthermore, we have the four fermionic spin- $\frac{1}{2}$ currents

$$
\begin{aligned}
Q_{+K}= & -\frac{1}{2} j_{B R S T}-2 Q^{2} \xi-Q \bar{\psi}_{l} \gamma_{-}-Q \psi_{l} \gamma_{+}-\frac{1}{4} \beta_{-} \gamma_{+} c+ \\
& +\frac{1}{4} \beta_{+} \gamma_{-} c-Q c \partial \phi_{l}-Q c \partial \bar{\phi}_{l}+\frac{1}{2} \gamma_{+} \gamma_{-} \eta+\frac{1}{4} c \partial c \eta \\
Q_{-K}= & \frac{1}{2} \eta \\
Q_{+}= & -\frac{1}{2} \jmath_{\text {ghost }}+Q \bar{\psi}_{l}, \\
Q_{-}= & +\frac{1}{2} \jmath_{\text {ghost- }}-Q \psi_{l} .
\end{aligned}
$$


and, finally, a spin-0 bosonic current

$$
\varphi=\frac{1}{2} \jmath_{g h o s t}-Q \phi_{l}-Q \bar{\phi}_{l}
$$

where the $U(1)$ current of the twisted $N=4$ superconformal algebra is given by $U=\partial \varphi$.

In the remainder of this section we will show how one can construct these currents by means of quantum Hamiltonian reduction of $\operatorname{ssp}(4 \mid 2)$. The bosonic part of this Lie superalgebra consists of an $s \ell(2)$ algebra at level $\kappa$ and an $s o(4)$ algebra at level $-2 \kappa$. We make use of the fact that $s o(4)$ can be written in terms of two commuting $s \ell(2)$ algebras, in redefining the currents

$$
\begin{aligned}
M^{ \pm} & =i\left(J^{12}+J^{34} \pm i J^{13} \mp i J^{23}\right), \\
M^{0} & =i\left(J^{14}+J^{23}\right), \\
N^{ \pm} & =i\left(-J^{12}+J^{34} \mp i J^{13} \pm i J^{23}\right), \\
N^{0} & =-i\left(J^{14}-J^{23}\right) .
\end{aligned}
$$

For the fermionic currents of $\operatorname{osp}(4 \mid 2)$, we choose a new basis as well,

$$
j^{ \pm a b}=\sigma_{i}^{a b} j^{ \pm i} \quad, \quad a, b \in\{+,-\}
$$

where

$$
\sigma_{1}=\left(\begin{array}{cc}
1 & 0 \\
0 & 1
\end{array}\right), \quad \sigma_{2}=\left(\begin{array}{cc}
0 & 1 \\
1 & 0
\end{array}\right), \quad \sigma_{3}=\left(\begin{array}{cc}
0 & -i \\
i & 0
\end{array}\right), \quad \sigma_{4}=\left(\begin{array}{cc}
1 & 0 \\
0 & -1
\end{array}\right)
$$

These fermionic currents transform under the representation $\left(\frac{1}{2}, \frac{1}{2}, \frac{1}{2}\right)$ of the three $s \ell(2)$ sub-algebras of $\operatorname{osp}(4 \mid 2)$.

We now define the gradation by choosing the Cartan element $H=E^{0}+M^{0}-N^{0}$. Accordingly, the algebra osp $(4 \mid 2)$ decomposes into positive, zero and negative parts, which are displayed in the following table:

\begin{tabular}{|c||c|c|c||c|c|c||c|c|c|}
\hline & $E^{+}$ & $E^{0}$ & $E^{-}$ & $M^{+}$ & $M^{0}$ & $M^{-}$ & $N^{+}$ & $N^{0}$ & $N^{-}$ \\
\hline$H$ & 1 & 0 & -1 & 1 & 0 & -1 & 1 & 0 & -1 \\
\hline
\end{tabular}

\begin{tabular}{|l||c|c|c|c|c|c|c|c|}
\hline & $j^{+++}$ & $j^{++-}$ & $j^{+-+}$ & $j^{+--}$ & $j^{-++}$ & $j^{-+-}$ & $j^{--+}$ & $j^{---}$ \\
\hline$H$ & $1 / 2$ & $3 / 2$ & $-1 / 2$ & $1 / 2$ & $-1 / 2$ & $1 / 2$ & $-3 / 2$ & $1 / 2$ \\
\hline
\end{tabular}


The constraints that we choose are compatible with the gradation and are given by:

$$
\begin{array}{r}
\Phi_{E}^{-}=E^{-}-\frac{\kappa}{2}, \\
\Phi_{M}^{-}=M^{-}-\mu, \\
\Phi_{N}^{+}=N^{+}-\nu, \\
\Phi^{+-}=j^{+-+}-\psi, \\
\Phi^{-++}=j^{-++}-\chi-\frac{1}{4} \bar{\psi} \nu, \\
\Phi^{--+}=j^{-++}, \\
\Phi^{--}=j^{---}-\bar{\chi}-\frac{1}{4} \bar{\psi} \mu,
\end{array}
$$

where

$$
\chi(z) \bar{\chi}(w)=-\frac{1}{4} \frac{\kappa}{(z-w)}, \quad \psi(z) \bar{\psi}(w)=\mu(z) \bar{\mu}(w)=\nu(z) \bar{\nu}(w)=\frac{1}{(z-w)} .
$$

As for the $N=1$ string, the $s \ell(2)$ embedding is given by $E^{ \pm}, E^{0}$, and we again face the problem of having highest weights in the negatively graded part. In the above choice of constraints, we took care of this by the introduction of the auxiliary fields $\mu, \bar{\mu}, \nu, \bar{\nu}, \psi, \bar{\psi}$; in addition, the auxiliary fields $(\chi, \bar{\chi})$ are needed to make the constraints first class. The corresponding gauge invariant action has the form

$$
S=S_{W Z W}[g]+\frac{1}{2 \pi} \int d^{2} z \operatorname{str}(\bar{A} \Phi)-\frac{1}{2 \pi} \int d^{2} z(\bar{\mu} \bar{\partial} \mu+\bar{\nu} \bar{\partial} \nu+\bar{\psi} \bar{\partial} \psi)-\frac{2}{\kappa \pi} \int d^{2} z \bar{\chi} \bar{\partial} \chi
$$

Accordingly, the gauge transformation for the fields are

$$
\begin{array}{rlrl}
\delta \chi=\frac{\kappa}{2} \eta^{+++}, \delta \bar{\chi}=\frac{\kappa}{2} \eta^{+--}, & & \\
\delta \psi=-\frac{1}{2} \eta^{+++} \mu-\frac{1}{2} \eta^{+--} \nu, & & \delta \bar{\psi}=2 \eta^{-+-}, \\
\delta \mu=0, & \delta \bar{\mu}=-\eta_{M}^{+}+\frac{1}{2} \eta^{+++} \psi, \\
\delta \nu=0, & \delta \bar{\nu}=-\eta_{N}^{-}+\frac{1}{2} \eta^{+++} \psi, \\
\delta \bar{A}=\bar{\partial} \eta+[\bar{A}, \eta], & \delta J=\partial \eta+[J, \eta] .
\end{array}
$$

We do the gauge fixing analogous to the previous chapter. In order to obtain the BRST operator, we need the following ghosts (these are not to be confused with the ghosts of the $N=2$ system !):

$$
\begin{aligned}
& C=c e_{+}+c^{M} t_{+}^{M}+c^{N} t_{-}^{N}+\gamma^{-+-} t_{-+-}+\gamma^{+--} t_{+--}+\gamma^{++-} t_{++-}+\gamma^{+++} t_{+++}, \\
& B=b e_{-}+b^{M} t_{-}^{M}+b^{N} t_{+}^{N}+\beta^{+-+} t_{+-+}+\beta^{-++} t_{-++}+\beta^{--+} t_{--+}+\beta^{---} t_{---} .
\end{aligned}
$$


From the ghost action, which is the same as (2.7), we can read off the OPE's for the ghosts:

$$
\begin{array}{r}
b(z) c(w)=\frac{1}{(z-w)}, \quad b^{M}(z) c^{M}(w)=b^{N}(z) c^{N}(w)=\frac{-2}{(z-w)}, \\
\beta^{+-+}(z) \gamma^{-+-}(w)=\beta^{-++}(z) \gamma^{+--}(w)=\beta^{--}(z) \gamma^{+++}(w)= \\
=-\beta^{--+}(z) \gamma^{++-}(w)=\frac{1}{(z-w)} .
\end{array}
$$

The currents $J$ of $\operatorname{osp}(4 \mid 2)$ get modified by the ghost contributions: $J \rightarrow \hat{J} \equiv J+J_{g h}$, where

$$
\begin{aligned}
E_{g h}^{+} & =\frac{1}{2} \beta^{+-+} \gamma^{++-} \\
E_{g h}^{0} & =-\frac{1}{2} b c-\frac{1}{4} \beta^{+-+} \gamma^{-+-}+\frac{1}{4} \beta^{-++} \gamma^{+--}-\frac{1}{4} \beta^{--+} \gamma^{++-}+\frac{1}{4} \beta^{---} \gamma^{+++} \\
E_{g h}^{-} & =-\frac{1}{2} \beta^{--+} \gamma^{-+-} \\
M_{g h}^{+} & =\frac{1}{2} \beta^{-++} \gamma^{++-} \\
M_{g h}^{0} & =\frac{1}{4} b^{M} c^{M}+\frac{1}{4} \beta^{+-+} \gamma^{-+-}-\frac{1}{4} \beta^{-++} \gamma^{+--}-\frac{1}{4} \beta^{--+} \gamma^{++-}+\frac{1}{4} \beta^{---} \gamma^{+++} \\
M_{g h}^{-} & =-\frac{1}{2} \beta^{--+} \gamma^{+--} \\
N_{g h}^{+} & =-\frac{1}{2} \beta^{--+} \gamma^{+++} \\
N_{g h}^{0} & =-\frac{1}{4} b^{N} c^{N}-\frac{1}{4} \beta^{+-+} \gamma^{-+-}-\frac{1}{4} \beta^{-++} \gamma^{+--}+\frac{1}{4} \beta^{--+} \gamma^{++-}+\frac{1}{4} \beta^{---} \gamma^{+++}, \\
N_{g h}^{-} & =-\frac{1}{2} \beta^{--+} \gamma^{-+-} \\
j_{g h}^{+++} & =-\frac{1}{4} b^{N} \gamma^{++-}-\frac{1}{4} \beta^{+-+} c^{M}+\frac{1}{2} \beta^{-++} c \\
j_{g h}^{+-+} & =-\frac{1}{4} b^{M} \gamma^{+++}-\frac{1}{4} b^{N} \gamma^{+--}+\frac{1}{2} \beta^{--+} c \\
j_{g h}^{+--} & =-\frac{1}{4} b^{M} \gamma^{++-}-\frac{1}{4} \beta^{+-+} c^{N}+\frac{1}{2} \beta^{---} c \\
j_{g h}^{-++} & =\frac{1}{2} b \gamma^{+++}-\frac{1}{4} b^{N} \gamma^{-+-}-\frac{1}{4} \beta^{--+} c^{M} \\
j_{g h}^{-+-} & =\frac{1}{2} b \gamma^{++-}-\frac{1}{4} \beta^{-++} c^{N}-\frac{1}{4} \beta^{---} c^{M} \\
j_{g h}^{---} & =\frac{1}{2} b \gamma^{+--}-\frac{1}{4} b^{M} \gamma^{-+-}-\frac{1}{4} \beta^{--+} c^{N} \\
& =0
\end{aligned}
$$

The BRST operator can be split according to our gradation as $\mathcal{Q}_{B R S T}=\mathcal{Q}_{0}+\mathcal{Q}_{1}+\mathcal{Q}_{2}$, where

$\mathcal{Q}_{0}=\frac{1}{2 \pi i} \oint d^{2} z\left[-\frac{\kappa}{2} c+c^{M} \mu+c^{N} \nu\right]$, 


$$
\begin{aligned}
\mathcal{Q}_{1}= & \frac{1}{2 \pi i} \oint d^{2} z\left[-\gamma^{-+-} \psi-\gamma^{+--}\left(\chi-\frac{1}{4} \bar{\psi} \nu\right)-\gamma^{+++}\left(\bar{\chi}-\frac{1}{4} \bar{\psi} \mu\right)\right], \\
\mathcal{Q}_{2}= & \frac{1}{2 \pi i} \oint d^{2} z\left[c\left(E^{-}+\frac{1}{2} E_{g h}^{-}\right)-c^{M}\left(M^{-}+\frac{1}{2} M_{g h}^{-}\right)-c^{N}\left(N^{-}+\frac{1}{2} N_{g h}^{-}\right)-\gamma^{-+-}\left(j^{+-+}+\right.\right. \\
& \left.\left.+\frac{1}{2} j_{g h}^{+-+}\right)-\gamma^{+--}\left(j^{-++}+\frac{1}{2} j_{g h}^{-++}\right)+\gamma^{++-} j^{--+}-\gamma^{+++}\left(j^{---}+\frac{1}{2} j_{g h}^{---}\right)\right] . \quad(3.49)
\end{aligned}
$$

Analogously to the $N=1$ string, we can now solve the BRST cohomology and arrive at a realization of the non-linear $N=4$ superconformal algebra. Since the explicit expressions are rather lengthy, we state only the correspondence of the $s \ell(2)$ highest weights with the generators of the $N=4$ algebra:

$$
\begin{aligned}
& \hat{E}^{+} \rightarrow T, \\
& \hat{M}^{i} \rightarrow A^{+i}, \quad \hat{N}^{i} \rightarrow A^{-i}, \\
& \hat{j}^{+++} \rightarrow G_{+} \quad, \quad \hat{j}^{+--} \rightarrow G_{-}, \\
& \hat{j}^{++-} \rightarrow G_{+K} \quad, \quad \psi \rightarrow G_{-K} .
\end{aligned}
$$

After the Miura transformation, we get a free-field realization in terms of the Cartan generators and the auxiliary fields. To linearize the algebra, we adjoin four fermions and a $U(1)$ current with operator products

$$
\rho(z) \bar{\rho}(w)=\tau(z) \bar{\tau}(w)=\frac{\kappa}{(z-w)}, \quad u(z) u(w)=\frac{2 \kappa}{(z-w)^{2}} .
$$

The necessary redefinitions of the generators are given in (B.8). We take here $(\rho, \bar{\rho})$ corresponding to $\left(Q_{+}, Q_{-}\right)$and $(\tau, \bar{\tau})$ corresponding to $\left(Q_{+K}, Q_{-K}\right)$. The final form of the generators can now be fixed by requiring that they match the form of the twisted $N=4$ currents of the $N=2$ string. This leads to the following similarity transformations:

$$
\begin{aligned}
S= & \exp \left[\frac { 1 } { 2 \pi i } \oint d z \left(-\frac{1}{\kappa} \bar{\psi} \chi \mu-\frac{1}{\kappa} \bar{\psi} \bar{\chi} \nu-\frac{1}{2 \kappa} \bar{\psi} \hat{M}^{0} \bar{\rho}-\frac{1}{\kappa} \bar{\psi} \hat{N}^{0} \bar{\rho}+\frac{1}{4 \kappa} \bar{\psi} u \bar{\rho}+\frac{1}{2 \kappa} \bar{\psi} \mu \tau+\right.\right. \\
& \left.\left.+\frac{1}{2 \kappa} \bar{\psi} \nu \bar{\tau}+\frac{1}{\kappa^{2}} \bar{\psi} \chi \bar{\chi} \bar{\rho}-\frac{1}{4 \kappa^{2}} \bar{\psi} \tau \bar{\tau} \bar{\rho}+\frac{1}{4 \kappa} \bar{\psi} \mu \bar{\mu} \bar{\rho}-\frac{1}{4 \kappa} \bar{\psi} \nu \bar{\nu} \bar{\rho}\right)\right] \\
R= & \exp \left[\frac{1}{2 \pi i} \oint d z\left(\frac{1}{2 \kappa} \bar{\mu} \tau \bar{\rho}-\frac{1}{2 \kappa} \bar{\mu} \bar{\tau} \bar{\rho}\right)\right] .
\end{aligned}
$$

which act on the currents as follows:

$$
\begin{aligned}
& \tilde{T}=R S T S^{-1} R^{-1}, \\
& \tilde{G}_{a}=R S G_{a} S^{-1} R^{-1}, \quad \tilde{A}^{ \pm i}=R S A^{ \pm i} S^{-1} R^{-1}, \\
& Q_{+}=R S \rho S^{-1} R^{-1}, \quad Q_{-}=R S \bar{\rho} S^{-1} R^{-1}, \\
& Q_{+K}=R S \tau S^{-1} R^{-1}, \quad Q_{-K}=R S \bar{\tau} S^{-1} R^{-1} \text {, } \\
& \tilde{u}=R S u S^{-1} R^{-1} .
\end{aligned}
$$


(Note that $R$ and $S$ do not commute with each other.) Finally, we bosonize the Cartan currents

$$
\begin{array}{r}
\hat{E}^{0}=\frac{i \sqrt{\kappa}}{4}\left(\partial \phi_{1}+\partial \bar{\phi}_{1}\right), \\
\tilde{u}=i \sqrt{\kappa}\left(\partial \phi_{2}+\partial \bar{\phi}_{2}\right), \\
\hat{M}^{0}=\frac{i \sqrt{\kappa}}{4}\left(\partial \bar{\phi}_{1}-\partial \phi_{1}+\partial \phi_{2}-\partial \bar{\phi}_{2}\right), \\
\hat{N}^{0}=\frac{i \sqrt{\kappa}}{4}\left(\partial \bar{\phi}_{1}-\partial \phi_{1}-\partial \phi_{2}+\partial \bar{\phi}_{2}\right), \\
\text { where } \quad \partial \phi_{i}(z) \partial \bar{\phi}_{j}(w)=-\frac{\delta_{i j}}{(z-w)^{2}},
\end{array}
$$

and rescale the fields

$$
\begin{gathered}
\rho \rightarrow \frac{1}{\sqrt{\kappa}} \rho, \quad \bar{\rho} \rightarrow \frac{1}{\sqrt{\kappa}} \bar{\rho}, \\
\chi \rightarrow \frac{2 i}{\sqrt{\kappa}} \chi, \quad \bar{\chi} \rightarrow \frac{2 i}{\sqrt{\kappa}} \bar{\chi}, \\
\psi \rightarrow \frac{1}{2} \psi, \quad \bar{\psi} \rightarrow 2 \bar{\psi}, \\
\mu \rightarrow \frac{1}{2} \mu, \quad \bar{\mu} \rightarrow 2 \bar{\mu} \\
\nu \rightarrow \frac{1}{2} \nu, \quad \bar{\nu} \rightarrow 2 \bar{\nu} \\
\tau \rightarrow \frac{1}{2 \kappa} \tau, \quad \bar{\tau} \rightarrow 2 \bar{\tau} .
\end{gathered}
$$

The currents (3.53) then become precisely the currents of the BRST algebra of the $N=2$ string. Since these expressions are in general rather long, we give here just the most interesting one:

$$
\begin{aligned}
G_{+K}= & \bar{\psi}\left(-\partial \phi_{1} \partial \bar{\phi}_{1}-i \frac{\sqrt{\kappa}}{2}\left(\partial^{2} \phi_{1}+\partial^{2} \bar{\phi}_{1}\right)-\partial \phi_{2} \partial \bar{\phi}_{2}-\frac{1}{2} \chi \partial \bar{\chi}-\frac{1}{2} \bar{\chi} \partial \chi-\frac{1}{2} \rho \partial \bar{\rho}-\frac{1}{2} \bar{\rho} \partial \rho\right)- \\
& -i \frac{\sqrt{\kappa}}{2} \partial \bar{\psi}\left(\partial \phi_{2}-\partial \bar{\phi}_{2}\right)+\bar{\nu}\left(\frac{1}{2} \partial \phi_{1} \chi+\frac{1}{2} \partial \phi_{2} \rho+i \frac{\sqrt{\kappa}}{2} \partial \chi\right)-i \frac{\sqrt{\kappa}}{2} \partial \bar{\nu} \rho+\bar{\mu}\left(\frac{1}{2} \partial \bar{\phi}_{1} \bar{\chi}-\right. \\
& \left.-\frac{1}{2} \partial \bar{\phi}_{2} \bar{\rho}+i \frac{\sqrt{\kappa}}{2} \partial \bar{\chi}\right)-i \frac{\sqrt{\kappa}}{2} \partial \bar{\mu} \bar{\rho}+\tau\left(-\bar{\chi} \chi-\bar{\rho} \rho+i \frac{\sqrt{\kappa}}{2}\left(\partial \phi_{1}+\partial \bar{\phi}_{1}+\partial \phi_{2}-\partial \bar{\phi}_{2}\right)\right)+ \\
& +\tau \bar{\mu} \mu+\frac{1}{2} \bar{\mu} \partial \bar{\nu} \bar{\tau}-\frac{1}{2} \partial \bar{\mu} \bar{\nu} \bar{\tau}-\tau \bar{\nu} \nu-\psi \bar{\mu} \bar{\nu}-\bar{\psi} \psi \partial \bar{\psi}-\bar{\psi} \tau \partial \bar{\tau}-\frac{1}{2} \bar{\psi} \bar{\mu} \partial \mu- \\
& -\frac{1}{2} \bar{\psi} \bar{\nu} \partial \nu+\frac{1}{2} \bar{\psi} \partial \bar{\mu} \mu+\frac{1}{2} \bar{\psi} \partial \bar{\nu} \nu-\partial \bar{\psi} \tau \bar{\tau}-\partial \bar{\psi} \bar{\mu} \mu+\partial \bar{\psi} \bar{\nu} \nu .
\end{aligned}
$$

Clearly (3.56) represents the BRST-operator of the $N=2$ string. The matter system is represented by $\phi_{1}, \bar{\phi}_{1}$ and by $\chi, \bar{\chi}$, the Liouville system by $\phi_{1}, \bar{\phi}_{1}$ and by $\rho, \bar{\rho}$. The 
ghost system turns out to be given by $\psi, \bar{\psi}$ for the diffeomorphism ghosts, $\mu, \bar{\mu}$ and $\nu, \bar{\nu}$ for their bosonic superpartners and $\tau, \bar{\tau}$ for the $U(1)$ ghosts. The background charge of the Liouville system that cancels the conformal anomaly is given by $Q=i \sqrt{\kappa}$, where, of course, $\kappa$ is the level of the $\operatorname{ssp}(4 \mid 2)$ WZW-model.

\section{$4 s \ell(2 \mid 1)$ Embeddings and the Gauge Invariant Action for General N}

We have shown in the previous chapters how one can obtain $N=1$ and $N=2$ strings by Hamiltonian reduction. We would now like to briefly discuss the general properties of this procedure for $\operatorname{osp}(N \mid 2)$ with arbitrary $N$.

As mentioned above, one expects that $N$-2-extended string theories will be obtained by embeddings of $s \ell(2 \mid 1)$ into $\operatorname{ssp}(N \mid 2)$. Under the adjoint action of this embedding, the latter algebra will decompose into irreducible representations of $s \ell(2 \mid 1)$. The bosonic part of $s \ell(2 \mid 1)$ consists of an $s \ell(2)$ algebra $e_{ \pm}, e_{0}$ and a $\mathrm{U}(1)$-part $u$. The four fermionic generators $t_{ \pm}, \bar{t}_{ \pm}$transform as $\left(\frac{1}{2}, 1\right)$ and $\left(\frac{1}{2}, \overline{1}\right)$, respectively:

$$
\begin{aligned}
{\left[e_{0}, t_{ \pm}\right]= \pm \frac{1}{2} t_{ \pm} \quad\left[e_{0}, \bar{t}_{ \pm}\right] } & = \pm \frac{1}{2} \bar{t}_{ \pm} \\
{\left[u, t_{ \pm}\right]=t_{ \pm} } & {\left[u, \bar{t}_{ \pm}\right]=-\bar{t}_{ \pm} }
\end{aligned}
$$

Irreducible representations are labelled by two quantum numbers $(j, q)$, where $j$ is the spin and $q$ the $u$-charge. Regular irreducible representations span a multiplet



with $t_{+}|j, q\rangle=\bar{t}_{+}|j, q\rangle=0$. The embedding $s \ell(2 \mid 1) \hookrightarrow \operatorname{osp}(N \mid 2)$ is defined by the bosonic $s \ell(2)$ sub-algebra and by a Cartan element of the $s o(N)$-part. For the string- 
related Hamiltonian reduction, we require $q=0$ for all multiplets. This results into the following decomposition of $\operatorname{ssp}(N \mid 2)$ with respect to $\operatorname{s\ell }(2 \mid 1)$ :

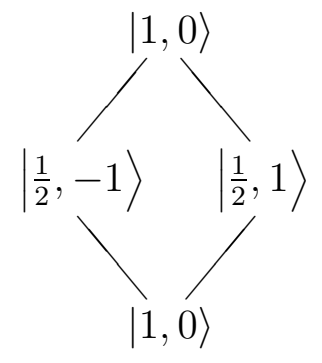

(1)

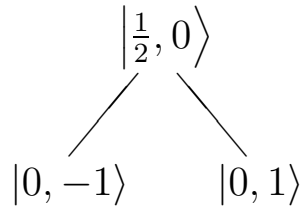

$(N-2)$ $|0,0\rangle$

$\left(\frac{1}{2} N(N-1)-2 N+3\right)$

The numbers below the multiplets denote the multiplicities. Of course, the $(j=1)$ multiplet corresponds precisely to the $s \ell(2 \mid 1)$ itself. It is fairly obvious that the string BRST current and the anti-ghost will arise from this multiplet, if we choose the gradation corresponding to $\left(e_{0}+u\right)$. From this it becomes clear that the $\mathrm{U}(1)$ part $u$ will represent the ghost number current of the string model. If we had chosen an embedding leading to multiplets with $q \neq 0$, these multiplets would have non-vanishing ghost number, and this appears not acceptable for a string theory.

The $N-2$ multiplets with $j=\frac{1}{2}$ will give rise to the underlying $N-2$ supersymmetries of the string. The states $|0,-1\rangle$ and $|0,1\rangle$ belong to the $\operatorname{so}(N)$ part of $\operatorname{osp}(N \mid 2)$. Note that there are in general $s \ell(2)$ highest weights that are negatively graded. These will give rise to bosonic auxiliary fields which correspond to the $N-2 \beta, \gamma$ systems of the string. Note also that the $j=\frac{1}{2}$ multiplets are short multiplets, which typically arise in the non-linear form of superconformal algebras.

Simple counting shows that we will have in addition $\frac{1}{2} N(N-1)-2 N+3$ singlets. They are simply the rest of the generators of the $s o(N)$ part. We note that due to this there will be an additional difficulty in the reduction for $N \geq 5$. Namely there will be generators of the $s o(N)$ part besides the Cartan elements that do not get automatically bosonized. Nevertheless, from cursory inspection we expect that one can find a bosonization of them that allows for a string interpretation.

In order to find gauge invariant lagrangian for general $\operatorname{ssp}(N \mid 2)$, let us first make 
a remark concerning different definitions of gradations. In the reduction process it is convenient to think of the gradation being defined on the current algebra. Thus the ghosts acquire non-trivial grades via to the ghost contribution to the currents, whereas the auxiliary fields have grade zero. This is important for finding the filtrations of BRST operators (2.13),(3.49). However, for finding the gauge invariant lagrangian, it is more convenient to define the gradation on the algebra itself. That is, we will assign non-trivial grades also to the auxiliary fields.

The starting point is the action

$$
S_{0}=-\frac{1}{2 \pi} \int d^{2} z \operatorname{str}\left(\frac{\kappa}{2} \bar{A} e_{-}+\bar{A} \psi+\bar{A} \varphi\right) .
$$

This is the a priori non-invariant part of the WZW-model on $\operatorname{osp}(N \mid 2)$, subject to the constraint that the lowering operator of $s \ell(2)$ is equal to $\frac{\kappa}{2}$. The field $\psi$ is the auxiliary field for the negatively graded highest weight of $s \ell(2 \mid 1)$ and $\varphi$ are the auxiliary fields for the negatively graded $s o(N)$ currents.

Under a gauge transformation we have ( $\eta$ being the gauge parameter)

$$
\begin{gathered}
\delta S_{0}=-\frac{1}{2 \pi} \int d^{2} z \operatorname{str}\left(\bar{A}_{\frac{1}{2}}\left(\frac{\kappa}{2}\left[\eta_{\frac{1}{2}}, e_{-}\right]+\left[\eta_{\frac{1}{2}}, \varphi_{-1}\right]\right)+\bar{\partial} \eta_{\frac{1}{2}} \bar{\psi}_{-\frac{1}{2}}+\bar{\partial} \eta_{1} \varphi_{-1}+\right. \\
\left.+\bar{A}_{\frac{1}{2}} \delta \psi_{-\frac{1}{2}}+\bar{A}_{1} \delta \varphi_{-1}\right)
\end{gathered}
$$

The subscripts denote explicitly the grades of the fields. Since under the decomposition $\operatorname{osp}(N \mid 2) \rightarrow s \ell(2 \mid 1)$ the half-integer graded fields can come only from the fermionic part, we can decompose $\bar{A}_{\frac{1}{2}}$ uniquely into $\hat{A}_{\frac{1}{2}} \in k e r a d_{e_{+}}$and $\tilde{A}_{\frac{1}{2}} \in k e r a d_{e_{-}}$. Let us now look at the term of the form $\operatorname{str}\left(\hat{A}_{\frac{1}{2}}\left[\eta_{\frac{1}{2}}, \varphi_{-1}\right]\right)$. We see that $\left[\eta_{\frac{1}{2}}, \varphi_{-1}\right]$ has to be an element of ker $a d_{e_{-}}$to have non-vanishing supertrace. Since $\varphi_{-1}$ lies in both $k e r a d_{e_{+}}$and $k e r a d_{e_{-}}$, this shows that only the part of $\eta_{\frac{1}{2}}$ contributes to this term which lies in ker $a d_{e_{+}}$. We denote this by $\tilde{\eta}_{\frac{1}{2}}$. Similar arguments lead us to introduce $\hat{\eta}_{\frac{1}{2}} \in \operatorname{ker} a d_{e_{-}}$. We arrive thus at

$$
\begin{gathered}
\delta S_{0}=-\frac{1}{2 \pi} \int d^{2} z \operatorname{str}\left(\hat{A}_{\frac{1}{2}}\left(\frac{\kappa}{2}\left[\tilde{\eta}_{\frac{1}{2}}, e_{-}\right]+\left[\hat{\eta}_{\frac{1}{2}}, \varphi_{-1}\right]\right)+\tilde{A}_{\frac{1}{2}}\left(\left[\tilde{\eta}_{\frac{1}{2}}, \varphi_{-1}\right]+\delta \psi_{-\frac{1}{2}}\right)+\right. \\
\left.+\bar{\partial} \hat{\eta}_{\frac{1}{2}} \psi_{-\frac{1}{2}}+\bar{\partial} \eta_{1} \varphi_{-1}+\bar{A}_{1} \delta \varphi_{-1}\right) .
\end{gathered}
$$

It is easy to see that gauge invariance can be restored by modifying the constraints, that is, by adding the following term to the lagrangian:

$$
S_{1}=-\frac{1}{2 \pi} \int d^{2} z \operatorname{str}\left(\tilde{A}_{\frac{1}{2}}\left(\left[\bar{\psi}_{\frac{1}{2}}, \varphi_{-1}\right]+\left[\chi, e_{-}\right]\right)\right)
$$


where the new field $\chi$ lies in the part of $\operatorname{osp}(N \mid 2)$ with half-integer grade. $\bar{\psi}_{\frac{1}{2}}$ is conjugate to $\psi_{-\frac{1}{2}}$ and therefore lies in $k e r a d_{e-}$. Gauge invariance also forces us to introduce kinetic terms for the auxiliary fields,

$$
S_{k i n}=-\frac{1}{2 \pi} \int d^{2} z \operatorname{str}\left(\bar{\partial} \bar{\psi}_{\frac{1}{2}} \psi_{-\frac{1}{2}}+\bar{\partial} \bar{\varphi}_{1} \varphi_{-1}+\frac{1}{\kappa} \bar{\partial} \chi\left[\chi, e_{-}\right]\right) .
$$

The whole action is gauge invariant if we assign to the auxiliary fields the following transformation rules:

$$
\begin{array}{ll}
\delta \varphi_{-1}=0 & , \delta \bar{\varphi}_{1}=\eta_{1}-\left[\tilde{\eta}_{\frac{1}{2}}, \bar{\psi}_{\frac{1}{2}}\right] \\
\delta \psi_{-\frac{1}{2}}=\hat{\eta}_{\frac{1}{2}} & , \quad \delta \psi_{-\frac{1}{2}}=-\left[\tilde{\eta}_{\frac{1}{2}}, \varphi_{-1}\right] \\
\delta \chi=\frac{\kappa}{2} \tilde{\eta}_{\frac{1}{2}} &
\end{array}
$$

From the gauge invariant action we can proceed in complete analogy to the previous sections, and infer the form of the constraints. The interpretation of the auxiliary fields is clear, in that $(\psi, \bar{\psi})$ will always correspond to the diffeomorphism ghosts of the string. Their bosonic superpartners can be identified with $(\varphi, \bar{\varphi})$. The additional ghosts that are present in string theories with extended supersymmetries will come in by linearizing the algebra. From our experience with the $N=1$ and $N=2$ strings we expect $\chi$ to describe the fermions in the matter sector.

\section{A Note on Topological Strings}

As pointed out, twisted $N=2$ algebras formed by the BRST-current, the anti-ghost together with the energy momentum tensor and the ghost current are one of the most basic features of the BRST formulation of string theories. The construction of topological strings 15 on the other hand involves from the very beginning realizations of twisted $N=2$ superconformal algebras for the matter, Liouville and ghost sector separately. The BRST operator of topological strings has the special property that it is the sum of $\mathcal{Q}_{s}=$ $\frac{1}{2 \pi} \oint G_{+} d z$ and $\mathcal{Q}_{v}=\frac{1}{2 \pi} \oint J_{B R S T}^{v} d z$, where $G_{+}$denotes the sum of the supercharges of the matter, Liouville and ghost systems and $J_{B R S T}^{v}$ is the BRST current that arises by gauge fixing the local world sheet symmetries. According to our general reasoning, we expect also for the topological string that $J_{B R S T}^{v}$ and $b$ form a twisted $N=2$ superconformal algebra. This suggests that there should actually be a doubly twisted $N=4$ superconformal algebra in the topological string. 
Let us first state some remarks on this hidden, doubly twisted $N=4$ superconformal algebra. We focus on the twisted currents

$$
\begin{aligned}
\hat{T} & =T+\partial M_{+-}+\partial M_{+K-K}+\frac{1}{2} \partial U=T+i \partial A^{+3}+\frac{1}{2} \partial U \\
\hat{G}_{+} & =G_{+}+\partial Q_{+}, \\
\hat{G}_{-} & =G_{-}+\partial Q_{-}, \\
\hat{G}_{+K} & =G_{+K}-\partial Q_{+K}, \\
\hat{G}_{-K} & =G_{-K}+\partial Q_{-K}, \\
\hat{J}_{1} & =2 i A^{+3} \\
\hat{J}_{2} & =i\left(A^{+3}-A^{-3}\right)+U .
\end{aligned}
$$

Note that the twist assigns conformal weight 1 to $\left\{G_{+}, G_{+K}\right\}$, weight 2 to $\left\{G_{-}, G_{-K}\right\}$, wight 0 to $A^{++}$, weight 2 to $A^{+-}$. The $\mathrm{U}(1)$-currents and $A^{--}$of the sub-algebra stay at conformal weight 1 .

At $k^{+}=k^{-}$the currents $\left\{\hat{T}, \hat{G}_{+}, \hat{G}_{-}, \hat{J}_{1}\right\}$ form a topologically twisted $N=2$ subalgebra with central extension $6 k^{+}$, whereas $\left\{\hat{T}, \hat{G}_{+K}, \hat{G}_{-K}, \hat{J}_{2}\right\}$ form a topologically twisted $N=2$ sub-algebra with vanishing central extension. It is quite remarkable that (5.1) together with $\left\{A^{++}, A^{+-}, A^{--}, Q_{-}, Q_{+K}\right\}$ form a closed sub-algebra. In the following we will see that it is exactly this sub-algebra that is realized in the topological string.

In the topological string we take the matter sector to be completely arbitrary. We only require that it exhibits a twisted $N=2$ superconformal symmetry, with some (twisted) central extension $c_{m}$. In representing the Liouville sector we follow [15] and introduce two bosonic fields $\{\pi, \varphi\}$ and two fermions $\{\chi, \psi\}$ with OPE's

$$
\pi(z) \varphi(w)=-\ln (z-w), \quad \partial \chi(z) \psi(w)=\frac{1}{z-w} .
$$

The generators of the twisted $N=2$ superconformal algebra in the Liouville sector are then

$$
\begin{aligned}
J_{l} & =-\partial \chi \psi-Q \partial \varphi+Q \partial \pi, \\
G_{l+} & =\partial \pi \psi-Q \partial \psi, \\
G_{l-} & =-\partial \chi \partial \varphi+Q \partial^{2} \chi \\
T_{l} & =-\partial \pi \partial \varphi-\partial \chi \partial \psi+Q \partial^{2} \pi,
\end{aligned}
$$


where $Q$ is an arbitrary background charge, in terms of which the central extension is $c_{l}=3+6 Q^{2}$. Since only the $G_{-}$supercharge corresponds to a local gauge symmetry, we have for the ghosts the usual fermionic $(b, c)$ system for the diffeomorphisms, plus a bosonic $(\beta, \gamma)$ system as their superpartners, with

$$
b(z) c(w)=\beta(z) \gamma(w)=\frac{1}{z-w}
$$

(both ghost systems have spins $(2,-1)$ ). The $N=2$ generators in the ghost sector look

$$
\begin{aligned}
J_{g} & =2 \beta \gamma-b c \\
G_{g+} & =-b \gamma \\
G_{g-} & =-\partial \beta c-2 \beta \partial c \\
T_{g} & =-2 b \partial c-\partial b c+2 \beta \partial \gamma+\partial \beta \gamma,
\end{aligned}
$$

and give rise to a twisted central extension of $c_{g}=-9$.

We are free to add the following total derivatives to the BRST current,

$$
\begin{aligned}
J_{B R S T}^{v}= & c\left(T_{m}+T_{l}+\frac{1}{2} T_{g}\right)+\gamma\left(G_{m-}+G_{l-}+\frac{1}{2} G_{g-}\right)- \\
& \left(Q+\frac{c_{m}}{6 Q}\right) \partial(c \partial \pi+\gamma \partial \chi),
\end{aligned}
$$

and introduce the ghost current:

$$
I_{g}=-b c+\beta \gamma+\left(Q+\frac{c_{m}}{6 Q}\right) \partial \pi
$$

The improvement terms in $J_{B R S T}^{v}$ and in $I_{g}$ are such that $\left\{T_{t o t}, J_{B R S T}^{v}, b, I_{g h}\right\}$ indeed forms a twisted $N=2$ superconformal algebraf with vanishing central extension. Furthermore, we find that

$$
\begin{aligned}
A^{++} & =-i c\left(G_{m+}+G_{l+}+\frac{1}{2} G_{g+}\right)+i \gamma\left(J_{m}+J_{l}+\frac{1}{2} J_{g}\right)-i k \partial \gamma \\
A^{+3} & =-\frac{i}{2}\left(J_{m}+J_{l}+J_{g}\right) \\
A^{+-} & =-i \beta
\end{aligned}
$$

form an $s \ell(2)$ Kac-Moody algebra at level

$$
k=\frac{1}{6} c_{t o t}
$$

\footnotetext{
${ }^{2}$ This algebra with vanishing central charge is of course different and independent from the "constituent" $N=2$ algebras and is analogous to the $N=2$ algebra that arises in the bosonic string.
} 
where

$$
c_{t o t} \equiv c_{l}+c_{g}+c_{m}=6\left(Q^{2}-1\right)+c_{m}
$$

Together with

$$
\begin{aligned}
A^{--} & =i c\left(G_{m-}+G_{l-}+\frac{1}{2} G_{g-}\right)+i\left(Q+\frac{c_{m}}{6 Q}\right) \partial(\partial \chi c) \\
Q_{-} & =\frac{1}{2} \beta c+\frac{1}{2}\left(Q+\frac{c_{m}}{6 Q}\right) \partial \chi \\
Q_{+K} & =-\frac{1}{2} c\left(J_{m}+J_{l}+\frac{1}{2} J_{g}\right)+\frac{1}{2}\left(Q+\frac{c_{m}}{6 Q}\right)(\partial \chi \gamma+\partial \pi c)+\frac{1}{2} k \partial c
\end{aligned}
$$

we thus see that the above-mentioned sub-algebra of the hidden, doubly twisted $N=4$ algebra is indeed realized in the topological string, with

$$
k^{+}=k^{-}=k \text {. }
$$

Note that due to the topological nature of the theory, the BRST operator $\oint J_{B R S T}^{v}$ is nilpotent for any $c_{t o t}$, and thus there is, strictly speaking, no critical central charge for the topological string. Nevertheless, the "critical" case $c_{t o t}=0$ is distinguished in the BRST algebra in that the level (5.9) of the $s \ell(2)$ algebra vanishes and the $s \ell(2)$ raising generator $A^{++}(5.8)$ decouples from the gravitational descendant, $\partial \gamma$.

Note also that the similarity transformation $U$ that rotates the topological string into the matter picture [16] arises here naturally from a Kac-Moody current:

$$
U=\exp \left[\frac{1}{2 \pi} \oint d z A^{--}\right]
$$

It is thus nothing but an inner automorphism of the doubly twisted $N=4$ superconformal algebra.

Although the structure of the doubly twisted $N=4$ algebra seems to be superficially similar to what we found for the $N=2$ string, there is an important difference. We do not get here enough fermions such as to form the non-linear version of the algebra, and it is precisely the non-linear form that arises from Hamiltonian reduction of osp $(4 \mid 2)$. Therefore it is unclear at the moment how one can construct topological strings along the lines that we presented in the previous sections. We will leave this problem for future investigation. 


\section{Conclusions}

The presented results clearly point to a systematic treatment of crticial and non-crititcal string theories. Indeed the structure revealed in the studied cases suggests that a very large class of string theories could be obtained from those Hamiltonian reductions which give rise to extensions of the $N=2$ superconformal algebras. Such reductions correspond to embeddings of $s \ell(2 \mid 1)$ in super Lie algebra. In addition, the grading has to be chosen in such a way that the realization obtained is consistent with the BRST structure, (1.1).

Though the complete classification of $s l(2 \mid 1)$ embeddings in Lie superalgebras has been achieved [17], this program is not yet finished. All examples studied so far have as a common characteristic that in the decomposition of the adjoint representation of the super Lie algebra in terms of irreducible representations of the embedded $\operatorname{sl}(2 \mid 1)$, only typical representations with vanishing $U(1)$ charge occur. Exactly for such cases we believe that the presented techniques are sufficient to recover the non-critical string theory. On the other hand, for generic $s l(2 \mid 1)$ embeddings, non-trivial $U(1)$ charges and atypical representations can occur. At this moment it is not clear how to generalize the methods developed in this paper to cover such cases. Further work in this direction is under way.

Several other questions remain unanswered. We found a hidden, doubly twisted $N=4$ structure in topological gravity. An obvious question is whether topological gravity can be obtained from Hamiltonian reduction. This is a relevant question, as the construction of extensions of topological gravity, such as topological $W$ gravity, turns out to be technically extremely involved. A more systematic approach, based on Hamiltonian reduction would greatly facilitate this task. 


\section{A Conventions for $\operatorname{osp}(N \mid 2)$}

We summarize our conventions for the current algebra of $\operatorname{ssp}(N \mid 2)$ :

$$
\begin{aligned}
E^{0}(z) E^{0}(w) & =\frac{\kappa}{8} \frac{1}{(z-w)^{2}} \\
E^{+}(z) E^{-}(w) & =\frac{\kappa}{4} \frac{1}{(z-w)^{2}}+\frac{E^{0}(w)}{(z-w)} \\
E^{0}(z) E^{ \pm}(w) & = \pm \frac{1}{2} \frac{E^{ \pm}(w)}{(z-w)} \\
J^{m}(z) J^{n}(w) & =\frac{1}{8} \frac{\delta^{m n}}{(z-w)^{2}}-\frac{1}{4} \frac{\sqrt{2} f_{m n}{ }^{k} J^{k}(w)}{(z-w)} \\
E^{0}(z) j^{ \pm k}(w) & = \pm \frac{1}{4} \frac{j^{ \pm k}(w)}{(z-w)}, \\
E^{ \pm}(z) j^{\mp k}(w) & =\frac{1}{2} \frac{j^{ \pm k}(w)}{(z-w)}, \\
J^{m}(z) j^{ \pm k}(w) & =\frac{1}{4} \frac{\sqrt{2} \lambda_{m n}{ }^{ \pm n}(w)}{(z-w)} \\
j^{ \pm m}(z) j^{ \pm n}(w) & =\mp \frac{1}{4} \frac{{\frac{\delta}{m n} \cdot E^{ \pm}}_{(z-w)}}{(z-w)^{2}}+\frac{1}{4} \frac{\delta^{m n}}{(z-w)}+\frac{1}{4} \frac{\sqrt{2} \lambda_{m n}{ }^{k} J^{k}(w)}{(z-w)} \\
j^{-n}(w) & =\frac{\delta^{m n}}{(z)}
\end{aligned}
$$

Here $E^{0}, E^{ \pm}$denote the $s \ell(2)$ part, $J^{m}$ the $s o(N)$ part and $j^{ \pm k}$ are the fermionic currents. For the $s o(N)$ algebra we employ a double index notation where $m$ stands for $p q$ with

$1 \leq p<q \leq N$. The structure constants are given by $f_{m n}{ }^{k}=\left[\lambda^{m}, \lambda^{n}\right]$ with $\lambda^{p q}{ }_{r s}=$ $\frac{1}{\sqrt{2}}\left(\delta_{r}^{p} \delta_{s}^{q}-\delta_{s}^{p} \delta_{r}^{q}\right)$. The metric used in our computations is

$$
g_{+-}=-2, \quad g_{00}=-4, \quad g_{+m-n}=-4 \delta_{m n}, \quad g_{m n}=-4 \delta_{m n} .
$$

\section{B Superconformal algebras with $N=3,4$}

1. $N=3$

The $N=3$ superconformal algebra consists of the stress tensor, three supercharges, an $s o(3)$ Kac Moody algebra at level $k$ and an additional fermionic current $F$ with 
spin $\frac{1}{2}$. All the fields are primary with respect to the stress tensor. The nonvanishing operator products are

$$
\begin{aligned}
G^{a}(z) G^{b}(w) & =\frac{2 g^{a b} k}{(z-w)^{3}}+\frac{\epsilon^{a b}{ }_{c} K^{c}(w)}{(z-w)^{2}}+\frac{2 g^{a b} T(w)+2 \epsilon^{a b}{ }_{c} \partial K^{c}(w)}{(z-w)} \\
K^{a}(z) K^{b}(w) & =\frac{-g^{a b} k}{(z-w)^{2}}+\frac{\epsilon^{a b}{ }_{c} K^{c}(w)}{(z-w)} \\
K^{a}(z) G^{b}(w) & =\frac{-F(w)}{(z-w)^{2}}+\frac{\epsilon^{a b}{ }_{c} G^{c}}{(z-w)(w)} \\
G^{a}(z) F(w) & =\frac{-K^{a}(w)}{(z-w)} \\
F(z) F(w) & =\frac{-k}{(z-w)} .
\end{aligned}
$$

The components of the metric are $g^{+-}=1$ and $g^{33}=2$. The central charge is given by $c=3 k$. The fermion can be factorized by redefining the generators as follows:

$$
\begin{aligned}
\tilde{T} & =T-\frac{1}{2 k} F \partial F, \\
\tilde{G}^{a} & =G^{a}-\frac{1}{k} F K^{a} .
\end{aligned}
$$

This then leads to the non-linear form of the algebra.

2. $N=4$

The large $N=4$ superconformal Algebra consists of the stress tensor, four supercharges, two commuting $\operatorname{sl}(2)$ Kac Moody algebras at different levels $k^{+}, k^{-}$, four fermionic currents with spin $\frac{1}{2}$ and a $U(1)$ current. All the fields are primary, the rest of the non-vanishing operator products are

$$
\begin{aligned}
G_{a}(z) G_{b}(w) & =\frac{2 c}{3} \frac{\delta_{a b}}{(z-w)^{3}}+\frac{2 M_{a b}(w)}{(z-w)^{2}}+\frac{2 T(w) \delta_{a b}+\partial M_{a b}(w)}{(z-w)} \\
A^{ \pm i}(z) G_{a}(w) & =\frac{\alpha^{ \pm i}{ }_{a}{ }^{b} G_{b}(w)}{z-w} \mp \frac{\frac{2 k^{ \pm}}{k} \alpha^{ \pm i}{ }_{a}{ }^{b} Q_{b}(w)}{(z-w)^{2}} \\
A^{ \pm i}(z) A^{ \pm j}(w) & =\frac{f^{i j}{ }_{k} A^{ \pm k}(w)}{(z-w)}-\frac{\frac{k^{ \pm}}{2} \delta^{i j}}{(z-w)^{2}} \\
Q_{a}(z) G_{b}(w) & =\frac{2\left(\alpha_{a b}^{+i} A_{i}^{+}(w)-\alpha_{a b}^{-i} A_{i}^{-}(w)\right)+\delta_{a b} U(w)}{(z-w)} \\
A^{ \pm i}(z) Q_{a}(w) & =\frac{\alpha^{ \pm i}{ }_{a}{ }^{b} Q_{b}(w)}{(z-w)}
\end{aligned}
$$




$$
\begin{aligned}
U(z) G_{a}(w) & =\frac{Q_{a}(w)}{(z-w)^{2}}, \\
Q_{a}(z) Q_{b}(w) & =-\frac{k}{2} \frac{\delta_{a b}}{(z-w)}, \\
U(z) U(w) & =-\frac{k}{2} \frac{(z-w)^{2}}{(z)}
\end{aligned}
$$

with central charge $c=\frac{6 k^{+} k^{-}}{k}, k=k^{+}+k^{-}$, and

$$
M_{a b}(z)=-\frac{4}{k}\left(k^{-} \alpha_{a b}^{+i} A_{i}^{+}(z)+k^{+} \alpha_{a b}^{-i} A_{i}^{-}(z)\right) .
$$

The index $i$ takes the values $\{+,-, 3\}$ and $\delta_{i j}$ and $f^{i j}{ }_{k}$ are defined as

$$
\begin{aligned}
\delta_{+-} & =\frac{1}{2}, \\
f^{+-}{ }_{3} & =-2 i \\
f_{ \pm}^{3 \pm} & =\mp i .
\end{aligned}
$$

The non zero values for $\delta_{a b}$ and $\alpha_{a b}^{ \pm i}=-\alpha_{b a}^{ \pm i}$ with $a, b$ running over $\{+,-,+K,-K\}$ are given by

$$
\begin{array}{cc}
\delta_{+-}=\frac{1}{2}, & \delta_{+K-K}=\frac{1}{2}, \\
\alpha_{+-}^{ \pm 3}=-\frac{i}{4}, & \alpha_{+K-K}^{ \pm 3}=\mp \frac{i}{4}, \\
\alpha_{++K}^{+-}=\frac{i}{2}, & \alpha_{--K}^{++}=-\frac{i}{2}, \\
\alpha_{-+K}^{-+}=-\frac{i}{2}, & \alpha_{+-K}^{--}=\frac{i}{2} .
\end{array}
$$

To get the non-linear form, one factorizes the fermions and the U(1) current by redefining the generators as follows:

$$
\begin{aligned}
\tilde{T} & =T+\frac{1}{k}\left(U U+\partial Q^{a} Q_{a}\right) \\
\tilde{G}_{a} & =G_{a}+\frac{2}{k} U G_{a}-\frac{2}{3 k^{2}} \epsilon_{a b c d} Q^{b} Q^{c} Q^{d}+\frac{4}{k} Q^{b}\left(\alpha_{b a}^{+i} \tilde{A}_{i}^{+}-\alpha_{b a}^{-i} \tilde{A}_{i}^{-}\right) \\
\tilde{A}^{ \pm i} & =A^{ \pm i}-\frac{1}{k} \alpha_{a b}^{ \pm i} Q^{a} Q^{b} .
\end{aligned}
$$

The four index antisymmetric tensor is given by

$$
\epsilon^{a b c d}= \pm\left(4 \alpha^{ \pm i a b} \alpha^{ \pm}{ }_{i}{ }^{c d}-\delta^{a c} \delta^{b d}+\delta^{a d} \delta^{b c}\right) .
$$




\section{References}

[1] B. Gato-Rivera and A. M. Semikhatov Phys. Lett. B293 (1992)

[2] M. Bershadsky, W. Lerche, D. Nemeschansky and N. P. Warner, Nucl. Phys. B401 (1993) 304

[3] N. Berkovits and C. Vafa, Mod. Phys. Lett. A9 (1994) 653

[4] K. Ito and H. Kanno, Hamiltonian Reduction and Topological Conformal Algebra in $c \leq 1$ Non-critical Strings, preprint UTHEP-264; Lie Superalgebra and Extended Topological Conformal Symmetry in Non-critical $W_{3}$ Strings, preprint UTHEP-277

[5] J. Gomis and H. Suzuki, Phys. Lett. B278 (1992) 266

[6] A. Giveon and M. Rocek, Nucl. Phys. B400 (1993) 145

[7] A. Sevrin and W. Troost, Phys. Lett. B315 (1993) 304;

J. de Boer and T. Tjin, Comm. Math.Phys.160 (1994) 317

[8] A. Sevrin, K. Thielemans and W. Troost, Nucl.Phys. B407 (1993) 459, Phys. Rev. D48 (1993) 2789

[9] V. Knizhnik, Theor. Math. Phys. 66 (1986) 69

M. Bershadsky, Phys. Lett. B174 (1986)

[10] P. Goddard and A. Schwimmer, Phys. Lett. B214 (1986) 209

[11] M. Ademollo, L. Brink, A. D'Adda, R. D'Auria, E. Napolitano, S. Sciuto, E. del Guidice, P. Di Vecchia, S. Ferrara, F. Gliozzi, R. Musto and R. Pettorino, Phys. Lett. B62 (1976) 105;

M. Ademollo, L. Brink, A. D'Adda, R. D'Auria, E. Napolitano, S. Sciuto, E. del Guidice, P. Di Vecchia, S. Ferrara, F. Gliozzi, R. Musto, R. Pettorino and J.H. Schwarz, Nucl. Phys. B111 (1976) 77

[12] H. Ooguri and C. Vafa, Nucl. Phys. B361(1991) 469, Nucl. Phys. B367(1991) 83, Mod. Phys. Lett. A5 (1990) 1389

[13] M. Günaydin, J. L. Petersen, A. Taormina and A. Van Proeyen, Nucl. Phys. B293 (1992) 72 
[14] A. Sevrin, W. Troost and A. Van Proeyen, Phys. Lett. B208 (1988) 447

[15] E. Verlinde and H. Verlinde, Nucl. Phys. B352 (1991) 1234

R. Dijkgraaf, E. Verlinde and H. Verlinde, Nucl. Phys. B352 (1991) 59

[16] T. Eguchi, Y. Yamada and S.-K. Yang, Pys. Lett. B305 (1993) 235

[17] E. Ragoucy and P. Sorba, private communication. 